\title{
The Computational Meaning of Probabilistic Coherence Spaces
}

\author{
Thomas Ehrhard \\ Laboratoire PPS \\ Université Paris Diderot and CNRS \\ thomas.ehrhard@pps.jussieu.fr
}

\author{
Michele Pagani \\ Laboratoire LIPN \\ Université Paris 13 \\ michele.pagani@lipn.univ-paris13.fr
}

\author{
Christine Tasson \\ Laboratoire PPS \\ Université Paris Diderot, \\ christine.tasson@pps.jussieu.fr
}

\begin{abstract}
We study the probabilistic coherent spaces - a denotational semantics interpreting programs by power series with non negative real coefficients. We prove that this semantics is adequate for a probabilistic extension of the untyped $\lambda$-calculus: the probability that a term reduces to a head normal form is equal to its denotation computed on a suitable set of values. The result gives, in a probabilistic setting, a quantitative refinement to the adequacy of Scott's model for untyped $\lambda$-calculus.
\end{abstract}

\section{INTRODUCTION}

Most denotational models of functional languages and of the $\lambda$-calculus interpret types as domains and programs as continuous (or stable, strongly stable etc) functions. These morphisms carry qualitative informations about the programs they interpret, in the sense that the interpretation of a term does only say whether a given value is needed for producing a given result, but not how many times it is used. In [Gir88], Girard introduced a denotational semantics of typed and untyped lambda-calculus which is quantitative: in this model objects are sets, and a morphism from $X$ to $Y$ is similar to a power series in the sense that it involves monomials which contain exponents (natural numbers), the elements of $X$ being considered as formal indeterminates. The model is also quantitative in another sense: morphisms involve coefficients applied to such monomials and these coefficients are sets to be considered as possibly infinite coefficients.

The simplest non trivial object of this model is $1=\{\star\}$, the singleton set which can also be considered as a onedimensional space. A point (in the categorical sense) of this object consists of $\star$ equipped with a coefficient which is a set. A morphism from 1 to 1 is a power series with one parameter and sets as coefficients. In other words, it is a set-valued function defined on pairs $([\star, \ldots, \star], \star)$ where $[\star, \ldots, \star]$ (a multiset made of a finite number of occurrences of $\star$ ) should be considered as an exponent associated with the formal indeterminate $\star$. In accordance with this power series interpretation, there is a natural way of applying such a morphism to a point of 1 (a set) to obtain a point of 1 (a set): such morphisms can be seen as "functions" or more precisely as functors which can be characterized as those which preserve directed colimits and pullbacks (called normal functors by Girard). If the interpretation of a program of type $1 \rightarrow 1$ contains the pair $([\star, \star, \star], \star)$, this means that it can produce $\star$ using $\star$ three times. The coefficient of $([\star, \star, \star], \star)$ in this interpretation is much harder to interpret, and one of the purposes of this paper is precisely to shed some light on this issue, in the untyped case.

Fortunately, it is possible to build quantitative models, similar to Girard's quantitative model, but where coefficients remain finite (see [Ehr05] for instance) and for this purpose, one can use a probabilistic approach, as in the probabilistic coherence spaces (PCS), introduced in [Gir04] and further developed by the first author and Danos in [DE11]. In this setting, an object $\mathcal{X}$ is a pair of a countable set $X$, called web (the same set that interpret a type in the quantitative setting), together with a subset $\mathrm{P}(\mathcal{X})$ of $\left(\mathbb{R}^{+}\right)^{X}$ which obeys a closure property defined by means of a probabilistic duality (see Section III). Morphisms from $\mathcal{X}$ to $\mathcal{Y}$ can be seen as $Y$-indexed families of power series with indeterminates in $X$ and coefficients in $\mathbb{R}^{+}$. At ground types, PCSs have a direct probabilistic interpretation which is lost at higher type. The web of the PCS $\mathcal{I}$ interpreting integers is the set of natural numbers and $\mathrm{P}(\mathcal{I})$ is the set of all sub-probability distributions of natural numbers. In [DE11], a clear operational meaning is given to the elements of $\mathrm{P}(\mathcal{I})$. The authors define a probabilistic extension of PCF and prove that every closed term $M$ of integer type is interpreted by a vector $\llbracket M \rrbracket \in \mathrm{P}(\mathcal{I})$ so that the coefficient $\llbracket M \rrbracket_{n}$ associated with any natural number $n$ is equal to the probability that $M$ converges to the numeral $\underline{n}$. This is a probabilistic version of Plotkin's Adequacy Theorem for the Scott semantics of PCF headreduction [Plo77]. At higher types, this operational intuition is lost: the interpretations of terms are more complex than probability distributions and scalars greater than 1 appear. A model $\mathcal{D}$ of the untyped $\lambda$-calculus is also built in [DE11], but no such computational interpretation is given: the goal of this paper is to fulfill this gap.

The model $\mathcal{D}$ is built as the limit of a sequence $\left\{\mathcal{D}_{\ell}\right\}_{\ell \in \mathbb{N}}$ of PCSs, the first of which, $\mathcal{D}_{0}$, has an empty web. The web of $\mathcal{D}_{1}$ is a singleton, and our main result (Theorem 22) proves that, for any closed term $M$ of a natural probabilistic extension of the untyped $\lambda$-calculus, the probability that $M$ head-reduces to a head normal form is equal to the sum of the scalars of its interpretation on the elements of the web of $\mathcal{D}_{2}$ (which is infinite but has a very simple structure). This is a probabilistic refinement of the Adequacy Theorem for Scott's model $D_{\infty}$ according to which the semantics of an untyped 
$\lambda$-term is different from the bottom element iff its headreduction terminates [Hyl76]. Our proof adapts the PlotkinReynolds logical relation approach to this quantitative setting and follows a method introduced by Pitts [Pit], consisting in building a relation of formal approximation which satisfies the same recursive equation as $\mathcal{D}$.

The setting in which $\mathcal{D}$ is built is quite different from the category of Scott domains: our morphisms are power series with non-negative real coefficients. Nevertheless, the model $\mathcal{D}$ seems as canonical as Scott's $D_{\infty}$ : its construction is direct and natural, it is obviously a reflexive object and therefore a model of both $\beta$ and $\eta$ and, as we have shown, it has a nice operational adequacy property. This indicates that probabilistic lambda-calculi are interesting and expressive languages whose theory should be further studied and whose potential applications should be explored.

\section{Related works}

The standard approach to the semantics of probabilistic languages is based on the use of powerdomains. In such settings, types are interpreted by domains on which acts a probabilistic powerdomain monad $\mathcal{V}$. A probabilistic program of type $A \rightarrow B$ is interpreted as a continuous function $\llbracket A \rrbracket \rightarrow \mathcal{V}(\llbracket B \rrbracket)$ which maps a value of $A$ to a probability distribution of values of $B$. In [Jon89], [JP89], Jones get computational adequacy results in the typed and untyped cases for call-by-value reduction. Our approach is different in that we deal with much more concrete objects. They give rise to continuous domains natively endowed with the structure required for interpreting the probabilistic primitives of our programming language (no powerdomain monad is needed). Moreover, our morphisms are power series whereas, in the powerdomain approach, morphisms are arbitrary continuous maps, and this is another major difference. Finally, our model is a semantics of classical linear logic.

\section{Notations}

We write $\mathbb{N}$ for the set of all natural numbers. We identify multisets of elements of $A$ with functions $A \rightarrow \mathbb{N}$. If $m$ is such a multiset, we write $\operatorname{Supp}(m)$ for its support set $\{a \in$ $A$ s.t. $m(a) \neq 0\}$. A finite multiset is a multiset with a finite support. We write $\mathcal{M}_{\text {fin }}(A)$ for the set of all finite multisets of elements of $A$. The empty multiset is [] and $\uplus$ is the multiset union: $m \uplus m^{\prime}(a):=m(a)+m^{\prime}(a)$.

Let $J \subseteq I$ be sets of indexes. A vector $x \in\left(\mathbb{R}^{+}\right)^{I}$ is given by its values $x_{a}$ on indexes $a \in I$. We denote by $\left.x\right|_{J}$ the restriction of $x$ to $J$, which is a vector of $\left(\mathbb{R}^{+}\right)^{J}$. For every $i \in I$, let us denote $e_{i} \in\left(\mathbb{R}^{+}\right)^{I}$ the sequence of non negative real numbers equals to 0 everywhere but on the $i$-th index where it is equal to 1 .

\section{Probabilistic $\lambda$-CAlCUlus}

The set of probabilistic $\lambda$-terms is given by the following grammar (with $p \in[0,1]$ ):

$$
\Lambda^{+}: \quad M, N::=*|x| \lambda x \cdot M|(M N)| M+{ }_{p} N .
$$

$$
\begin{gathered}
\frac{(\lambda x \cdot M) N \stackrel{1}{\rightarrow} M\{N / x\}}{M+{ }_{p} N \stackrel{\mathrm{p}}{\rightarrow} M} \quad \frac{\stackrel{\mathrm{p}}{M+}{ }_{p} N \stackrel{1-\mathrm{p}}{\rightarrow} N}{\rightarrow} M^{\prime} \\
\frac{M \stackrel{\mathrm{p}}{\rightarrow} M^{\prime} M \text { not abstraction }}{M N . M \stackrel{\mathrm{p}}{\rightarrow} \lambda x \cdot M^{\prime}}
\end{gathered}
$$

Fig. 1. The head-reduction of $\Lambda^{+}$.

$$
\begin{aligned}
& \Delta M \stackrel{1}{\longrightarrow} M M \underset{\frac{2}{3}}{\stackrel{\frac{1}{3}}{\longrightarrow}}(\lambda y \cdot x) M \stackrel{1}{\longrightarrow} x \underset{\frac{2}{3}}{\longrightarrow} \lambda y . y \\
& \underset{\aleph_{1}}{\mathrm{\Theta}} \stackrel{1}{\longrightarrow} \stackrel{1}{\longrightarrow} M(\boldsymbol{\Theta} M) \stackrel{\frac{1}{3}}{\longrightarrow}(\lambda y . x)(\mathbf{\Theta} M) \stackrel{1}{\longrightarrow} x
\end{aligned}
$$

Fig. 2. Reduction trees of the terms $\Delta M$ and $\Theta M$, with $M=\lambda y \cdot x+\frac{1}{3}$ $\lambda y \cdot y$.

The term $*$ is a constant, considered as a closed term. Although it is used in the proofs, the main results (Section IV-B) still hold without having $*$ in the syntax. Also, the proofs and results of the sequel do not rely on $p$ being a real and one can impose that the probability $p$ is rational. We adopt the usual $\lambda$-calculus conventions as in [Bar84]. In particular, $\mathrm{FV}(M)$ is the set of the variables having free occurrences in $M$, and $\Lambda_{0}^{+}$ is the set of the closed terms. We use the following notation for terms useful to build examples:

$$
\boldsymbol{\Theta}=(\lambda x y . y(x x y))(\lambda x y . y(x x y)), \boldsymbol{\Delta}=\lambda x . x x, \boldsymbol{\Omega}=\Delta \Delta .
$$

We will consider only the head-reduction, i.e. the small step operational semantics defined in Figure 1. The notation $M \stackrel{\mathrm{p}}{\rightarrow}$ $N$ means that the term $M$ reduces in one step to the term $N$ with probability $p \in[0,1]$. As expected, the normal forms of this strategy are the head normal forms, i.e. the terms of the shape $\lambda x_{1} \ldots x_{\ell} \cdot M N_{1} \ldots N_{n}$, with $M$ either a variable or $*$ . The set of head normal forms is denoted by hnf, the letter $H$ will be ranged over hnf.

Figure 2 gives two examples of reduction tree. The reduction is non-deterministic since there are two rules associated with the random constructor. Remark that $L+{ }_{p} N$ intuitively expresses a superposition between $L$ and $N$, rather than an uncertain knowledge whether the term is $L$ or $N$. Figure 2, for example, shows that $\Delta\left(\lambda y . x+_{\frac{1}{3}} \lambda y \cdot y\right)$ reduces to $\lambda y . x$ (with probability $\left.\frac{2}{9}\right)$, in which case the random term $\lambda y . x+\frac{1}{3} \lambda y . y$ behaves once as $\lambda y . y$ and once as $\lambda y . x$.

We are interested in the probability that a given term reduces to a given head normal form after an arbitrary large (but finite) number of reduction steps. Computing such a probability is not trivial because of the presence of non-normalizing terms. For example, the probability that $\boldsymbol{\Theta}\left(\lambda y . x+_{\frac{1}{3}} \lambda y . y\right)$ 
reduces to $x$ must be, intuitively, the limit of $\frac{1}{3} \sum_{n=0}^{\infty} \frac{2^{n}}{3^{n}}$ (see Figure 2). In order to make precise such an intuition, we present the head-reduction as a Markov process over the set $\Lambda^{+}$, following [DE11].

We consider the set $\Lambda^{+}$as a set of states and the transition matrix $\operatorname{Red} \in[0,1]^{\Lambda^{+} \times \Lambda^{+}}$given by the following:

$$
\operatorname{Red}_{M, N}:= \begin{cases}\sum_{\pi \in \Pi_{M, N}} p_{\pi}, & \begin{array}{l}
\text { where } \Pi_{M, N} \text { is the set of } \\
\text { derivations } \pi \text { of } M \stackrel{\mathrm{p}_{\pi}}{\rightarrow} N \\
\text { according to Figure } 1
\end{array} \\
1 & \begin{array}{l}
\text { if } M=N \text { is a head normal } \\
\text { form, }
\end{array} \\
0 & \text { otherwise. }\end{cases}
$$

Notice that, fixed $M$ and $N, \Pi_{M, N}$ has at most two elements, as for example in the case $M=N+{ }_{p} N$. Red is a stochastic matrix (i.e. for all terms $M, \sum_{N \in \Lambda^{+}} \operatorname{Red}_{M, N}=1$ ), the value of $\operatorname{Red}_{M, N}$ intuitively describes the probability of evolving from the state $M$ to the state $N$ in one step.

A term $M$ is absorbing whenever $\operatorname{Red}_{M, M}=1$ : the absorbing states are those which are invariant under the transition matrix. Notice that the head normal forms are all absorbing, but there are absorbing terms which are not hnf, such as $\Omega$.

The $n$-th power $\operatorname{Red}^{n}$ of the matrix Red is a stochastic matrix on $\Lambda^{+}$(in case $n=0$, we have the identity matrix on $\Lambda^{+}$). Intuitively, the value of $\operatorname{Red}_{M, N}^{n}$ is the probability of evolving from the state $M$ to the state $N$ in exactly $n$ steps.

Proposition 1 ([DE11, Lemma 32]). Let $M \in \Lambda^{+}$and $H$ absorbing, the sequence $\left\{\operatorname{Red}_{M, H}^{n}\right\}_{n \in \mathbb{N}}$ is monotonic.

We thus define the following matrix in $[0,1]^{\Lambda^{+} \times \mathrm{hnf}}$ :

$$
\operatorname{Red}_{M, H}^{\infty}:=\sup _{n=0}^{\infty}\left(\operatorname{Red}_{M, H}^{n}\right)
$$

Intuitively, $\operatorname{Red}_{M, H}^{\infty}$ defines the probability that $M$ reaches a head normal form $H$ in an arbitrary number of steps. In particular, recalling the first example in Figure 2, we have $\operatorname{Red}_{\Delta M, x}^{n}=\frac{1}{3}$ if $n \geq 3$, otherwise it is 0 , so $\operatorname{Red}_{\Delta M, x}^{\infty}=\frac{1}{3}$. As for the second example, $\operatorname{Red}_{\Theta M, x}^{n}=\frac{1}{3} \sum_{i=0}^{k}\left(\frac{2}{3}\right)^{i}$, for $4(k+1) \leq n<4(k+2)$, hence $\operatorname{Red}_{\Theta M, x}^{\infty}=1$.

In general, notice that we always have $\operatorname{Red}_{M, H}^{\infty} \leq 1$, for any $M, H$. The following lemma gathers all the syntactical properties we need in the sequel.

Lemma 2. For all $M, M^{\prime} \in \Lambda^{+}, p \in[0,1]$,

1) if $M \stackrel{\mathrm{p}}{\rightarrow} M^{\prime}$, then $\forall L \in \Lambda^{+}, M\{L / x\} \stackrel{\mathrm{p}}{\rightarrow} M^{\prime}\{L / x\}$;

2) $\forall H \in \operatorname{hnf}, \operatorname{Red}_{M, H}^{\infty}=\sum_{N \in \Lambda^{+}} \operatorname{Red}_{M, N} \operatorname{Red}_{N, H}^{\infty}$;

3) if $M \stackrel{1}{\rightarrow} M^{\prime}$, then $\forall H \in \mathrm{hnf}, \forall L_{0}, \ldots, L_{n-1} \in \Lambda^{+}$, $\operatorname{Red}_{M L_{0} \ldots L_{n-1}, H}^{\infty}=\operatorname{Red}_{M^{\prime} L_{0} \ldots L_{n-1}, H}^{\infty}$;

4) $\sum_{H \in \text { hnf }} \operatorname{Red}_{M, H}^{\infty}=\sum_{H \in \text { hnf }} \operatorname{Red}_{M *, H}^{\infty}$.

Proof: 1 . By induction on a derivation of $M \stackrel{\mathrm{p}}{\rightarrow} M^{\prime}$.
2. By definition of $\operatorname{Red}^{\infty}$, we have $\operatorname{Red}_{M, H}^{\infty}=$ $\sup \left\{\operatorname{Red}_{M, H}^{0}, \sum_{N \in \Lambda^{+}} \operatorname{Red}_{M, N} \operatorname{Red}_{N, H}^{\infty}\right\}$. If $M \neq H$, the first quantity is 0 (so the sup is the second quantity); if $M=H$, both quantities are 1 , since $H$ is absorbing.

3. By induction on $n$. The base case is when $n=0$ or $M$ is not an abstraction, in this case $M L_{0} \ldots L_{n-1} \stackrel{1}{\rightarrow} M^{\prime} L_{0} \ldots L_{n-1}$, and the claim follows by item 2. Otherwise, $M=\lambda z \cdot N$, then $M^{\prime}=\lambda z \cdot N^{\prime}$ with $N \stackrel{1}{\rightarrow} N^{\prime}$. By item $1, N\left\{L_{0} / z\right\} \stackrel{1}{\rightarrow} N^{\prime}\left\{L_{0} / z\right\}$, hence by induction hypothesis $\operatorname{Red}_{N\left\{L_{0} / z\right\} L_{1} \ldots L_{n-1}, H}^{\infty}=$ $\operatorname{Red}_{N^{\prime}\left\{L_{0} / z\right\} L_{1} \ldots L_{n-1}, H}^{\infty}$. We conclude since by item 2, $\operatorname{Red}_{M L_{0} L_{1} \ldots L_{n-1}, H}^{\infty}=\operatorname{Red}_{N\left\{L_{0} / z\right\} L_{1} \ldots L_{n-1}, H}^{\infty}$ and $\operatorname{Red}_{M^{\prime} L_{0} L_{1} \ldots L_{n-1}, H}^{\infty}=\operatorname{Red}_{N^{\prime}\left\{L_{0} / z\right\} L_{1} \ldots L_{n-1}, H}^{\infty}$.

4. One splits depending whether or not $M$ is an abstraction. If $M$ is not an abstraction, then one proves, by induction on $n$, that $\sum_{H \in \text { hnf }} \operatorname{Red}_{M, H}^{n}=\sum_{H \in \text { hnf }} \operatorname{Red}_{M *, H}^{n}$, from which the claim follows. The base case is a consequence of: $M \in \mathrm{hnf}$ iff $M * \in \mathrm{hnf}$; the induction step is a consequence of: $M \stackrel{\mathrm{p}}{\rightarrow} M^{\prime}$ iff $M * \stackrel{\mathrm{p}}{\rightarrow} M^{\prime} *$.

If $M=\lambda z \cdot N$, one proves $\sum_{H \in \mathrm{hnf}} \operatorname{Red}_{\lambda z . N, H}^{n}=$ $\sum_{H \in \operatorname{hnf}} \operatorname{Red}_{N\{* / z\}, H}^{n}$, by induction on $n$. This equality entails $\sum_{H \in \text { hnf }} \operatorname{Red}_{\lambda z . N, H}^{\infty}=\sum_{H \in \mathrm{hnf}} \operatorname{Red}_{N\{* / z\}, H}^{\infty}$, and we conclude since $\operatorname{Red}_{(\lambda z . N) *, H}^{\infty}=\operatorname{Red}_{N\{* / z\}, H}^{\infty}$ by item 2 . The base case of the induction is a consequence of: $\lambda z \cdot N \in \mathrm{hnf}$ iff $N\{* / z\} \in \mathrm{hnf}$; the induction step follows from: $\lambda z . N \stackrel{\mathrm{p}}{\rightarrow} M^{\prime}$ iff $M^{\prime}=\lambda z \cdot N^{\prime}$ and $N\{* / z\} \stackrel{\mathrm{p}}{\rightarrow} N^{\prime}\{* / z\}$.

\section{Probabilistic Coherence Spaces}

In order to be self-contained, we shortly recall the notions and results of [DE11], but omit the proofs. After presenting the probabilistic coherence spaces, we sketch how they yield a model of linear logic (Section III-A) and of pure $\lambda$-calculus (Section III-B and III-C).

Let $I$ be a set, for any $x, y \in\left(\mathbb{R}^{+}\right)^{I}$, the pairing is defined as usual

$$
\langle x, y\rangle:=\sum_{i \in I} x_{i} y_{i} \in \mathbb{R}^{+} \cup\{\infty\}
$$

Given a set $\mathrm{P} \subseteq\left(\mathbb{R}^{+}\right)^{I}$ we define $\mathrm{P}^{\perp}$, the orthogonal of $\mathrm{P}$, as

$$
\mathrm{P}^{\perp}:=\left\{y \in\left(\mathbb{R}^{+}\right)^{I} \mid \forall x \in \mathrm{P} \quad\langle x, y\rangle \leq 1\right\} .
$$

Polar satisfies the following immediate properties: $\mathrm{P} \subseteq$ $\mathrm{P}^{\perp \perp}$, if $\mathrm{P} \subseteq \mathrm{Q}$ then $\mathrm{Q}^{\perp} \subseteq \mathrm{P}^{\perp}$, and then $\mathrm{P}^{\perp}=\mathrm{P}^{\perp \perp \perp}$.

Probabilistic coherence spaces are built around the notion of the interaction between programs and environments. Roughly speaking, both of them are interpreted as vectors in $\left(\mathbb{R}^{+}\right)^{I}$, for a suitable index set $I$, and their pairing gives a quantitative estimation of their interaction. The orthogonal operation is there to express the duality between programs and environments: its definition is such that the pairing between a vector associated with a program and a vector associated with an environment takes value in $[0,1]$, expressing the probability that the two succeed in interacting.

Definition 3 ([Gir04], [DE11]). A probabilistic coherence space, or PCS for short, is a pair $\mathcal{X}=(|\mathcal{X}|, \mathrm{P}(\mathcal{X}))$ where 
$|\mathcal{X}|$ is a countable set called the web of $\mathcal{X}$ and $\mathrm{P}(\mathcal{X})$ is a subset of $\left(\mathbb{R}^{+}\right)^{|\mathcal{X}|}$ such that the following holds:

closedness: $\mathrm{P}(\mathcal{X})^{\perp \perp}=\mathrm{P}(\mathcal{X})$,

boundedness: $\forall a \in|\mathcal{X}|, \exists \mu>0, \forall x \in \mathrm{P}(\mathcal{X}), x_{a} \leq \mu$,

completeness: $\forall a \in|\mathcal{X}|, \exists \lambda>0, \lambda e_{a} \in \mathrm{P}(\mathcal{X})$.

The dual of a PCS $\mathcal{X}$ is defined by $\mathcal{X}^{\perp}:=\left(|\mathcal{X}|, \mathrm{P}(\mathcal{X})^{\perp}\right)$.

Where recall that $e_{a}$ is the base vector for $a:\left(e_{a}\right)_{b}:=\delta_{a, b}$, with $\delta$ denoting the Kronecker delta. The boundedness condition requires the projection of $\mathrm{P}(\mathcal{X})$ in any direction to be bounded, while completeness sets $\mathrm{P}(\mathcal{X})$ to cover every direction. They have been introduced in [DE11] for keeping finite all the real numbers involved; they are not explicitly stated in the definition of PCS in [Gir04].

Notice that we do not require $\mathrm{P}(\mathcal{X}) \subseteq[0,1]^{|\mathcal{X}|}$, we shall understand why with the exponential construction.

It will be useful to consider $\left(\mathbb{R}^{+} \cup\{\infty\}\right)^{|\mathcal{X}|}$ as a partially ordered set, with the pointwise order:

$$
x \leq y:=\forall a \in|\mathcal{X}|, x_{a} \leq y_{a},
$$

with the lub of a set $P \subseteq\left(\mathbb{R}^{+} \cup\{\infty\}\right)^{|\mathcal{X}|}$ given pointwise: $\forall a \in|\mathcal{X}|,(\sup P)_{a}:=\sup _{x \in P} x_{a}$.

Proposition 4 ([DE11, Section 1.3]). For any PCS $\mathcal{X}, \mathrm{P}(\mathcal{X})$ is downward closed $(x \leq y \in \mathrm{P}(\mathcal{X}) \Rightarrow x \in \mathrm{P}(\mathcal{X}))$ and complete (if $D \subseteq \mathrm{P}(\mathcal{X})$ is directed, then $\sup D \in \mathrm{P}(\mathcal{X})$ ).

\section{A. Pcoh is a Model of Linear Logic}

We sketch the category Pcoh, showing that it yields a model of linear logic [Gir87], and then the cartesian closed category Pcoh! given by the cokleisly construction associated with the exponential comonad in Pcoh.

The objects of Pcoh are the PCSs and the $\operatorname{set} \operatorname{Pcoh}(\mathcal{X}, \mathcal{Y})$ of morphisms from $\mathcal{X}$ to $\mathcal{Y}$ is the set of those matrices $u \in\left(\mathbb{R}^{+}\right)^{|\mathcal{X}| \times|\mathcal{Y}|}$ such that $\forall x \in \mathrm{P}(\mathcal{X}), u \cdot x \in \mathrm{P}(\mathcal{Y})$, where $u \cdot x$ is the usual matricial product: $\forall b \in|\mathcal{Y}|$, $(u \cdot x)_{b}:=\sum_{a \in|\mathcal{X}|} u_{a, b} x_{a}$.

The identity $\mathrm{id}^{\mathcal{X}}$ on $\mathcal{X}$ is defined as the diagonal matrix given by $\left(\mathrm{id}^{\mathcal{X}}\right)_{a, a^{\prime}}=\delta_{a, a^{\prime}}$. The composition of morphisms is the matrix multiplication: $(u \circ v)_{a, c}=\sum_{b \in|\mathcal{Y}|} v_{a, b} u_{b, c}$, where $v \in \operatorname{Pcoh}(\mathcal{X}, \mathcal{Y}), u \in \operatorname{Pcoh}(\mathcal{Y}, \mathcal{Z})$, and $a \in|\mathcal{X}|, c \in|\mathcal{Z}|$. Such a sum converges in $\mathbb{R}^{+}$since $u$ and $v$ are Pcoh morphisms.

1) *-autonomous structure: The bifunctor $\otimes:$ Pcoh $\times$ $\mathbf{P c o h} \rightarrow \mathbf{P c o h}$ is defined by

$$
\begin{aligned}
|\mathcal{X} \otimes \mathcal{Y}| & :=|\mathcal{X}| \times|\mathcal{Y}| \\
\mathrm{P}(\mathcal{X} \otimes \mathcal{Y}) & :=\{x \otimes y \text { s.t. } x \in \mathrm{P}(\mathcal{X}), y \in \mathrm{P}(\mathcal{Y})\}^{\perp \perp},
\end{aligned}
$$

where $(x \otimes y)_{a, b}:=x_{a} y_{b}$, for $a \in|\mathcal{X}|$ and $b \in|\mathcal{Y}|$.

The action of $\otimes$ on morphisms $u \in \operatorname{Pcoh}(\mathcal{X}, \mathcal{Y})$ and $v \in$ $\operatorname{Pcoh}\left(\mathcal{X}^{\prime}, \mathcal{Y}^{\prime}\right)$ is defined by $(u \otimes v)_{\left(a, a^{\prime}\right),\left(b, b^{\prime}\right)}:=u_{a, b} v_{a^{\prime}, b^{\prime}}$, for $\left(a, a^{\prime}\right) \in\left|\mathcal{X} \otimes \mathcal{X}^{\prime}\right|,\left(b, b^{\prime}\right) \in\left|\mathcal{Y} \otimes \mathcal{Y}^{\prime}\right|$. The unity of $\otimes$ is given by the singleton web PCS $1:=\left(\{\star\},[0,1]^{\{\star\}}\right)$.

The object of linear morphisms $\mathcal{X} \multimap \mathcal{Y}$ is defined as

$$
\begin{aligned}
|\mathcal{X} \multimap \mathcal{Y}| & :=|\mathcal{X}| \times|\mathcal{Y}|, \\
\mathrm{P}(\mathcal{X} \multimap \mathcal{Y}) & :=\operatorname{Pcoh}(\mathcal{X}, \mathcal{Y}) .
\end{aligned}
$$

One proves that $\mathcal{X} \multimap \mathcal{Y}$ is a PCS by checking $\mathcal{X} \multimap \mathcal{Y}=$ $\left(\mathcal{X}^{\perp} \otimes \mathcal{Y}\right)^{\perp}$. The evaluation morphism ev $\in \operatorname{Pcoh}(\mathcal{X} \otimes(\mathcal{X} \multimap$ $\mathcal{Y}), \mathcal{Y})$ is given by $\operatorname{ev}_{\left(a,\left(a^{\prime}, b\right)\right), b^{\prime}}=\delta_{a, a^{\prime}} \delta_{b, b^{\prime}}$.

Last, the dualizing object $\perp$ is defined as the dual of $\mathbf{1}$ which is indeed equal to $1: \perp:=\mathbf{1}^{\perp}=\mathbf{1}$.

2) Cartesian structure: Pcoh admits the cartesian product of any countable family $\left(\mathcal{X}_{i}\right)_{i \in I}$ of PCSs, defined by

$$
\begin{aligned}
\left|\&_{i \in I} \mathcal{X}_{i}\right|: & =\cup_{i \in I}\left(\{i\} \times\left|\mathcal{X}_{i}\right|\right), \\
\mathrm{P}\left(\&_{i \in I} \mathcal{X}_{i}\right): & =\left\{x \in\left(\mathbb{R}^{+}\right)^{\left|\&_{i \in I} \mathcal{X}_{i}\right|} \text { s.t. } \begin{array}{l}
\forall i \in I, \\
\pi_{i}(x) \in \mathrm{P}\left(\mathcal{X}_{i}\right)
\end{array}\right\} .
\end{aligned}
$$

where $\pi_{i}(x)$ is the vector in $\left(\mathbb{R}^{+}\right)^{\left|\mathcal{X}_{i}\right|}$ denoting the $i$-th component of $x$, i.e. $\pi_{i}(x)_{a}:=x_{(i, a)}$. The $j$-th projection $\operatorname{pr}^{j} \in \operatorname{Pcoh}\left(\&_{i \in I} \mathcal{X}_{i}, \mathcal{X}_{j}\right)$ is defined by $\operatorname{pr}_{(i, a), b}^{j}:=\delta_{i, j} \delta_{a, b}$.

Notice that the empty product yields the terminal object of Pcoh. We may write $\mathcal{A}_{1} \& \mathcal{A}_{2}$ for the binary product: in the sequel, we can present any $x \in \mathrm{P}\left(\mathcal{A}_{1} \& \mathcal{A}_{2}\right)$ as the pair $\left(\pi_{1}(x), \pi_{2}(x)\right) \in \mathrm{P}\left(\mathcal{A}_{1}\right) \times \mathrm{P}\left(\mathcal{A}_{2}\right)$ of its components. When $\mathcal{X}_{i}$ is equal to $\mathcal{X}$ for each $i \in I$, we may write the product $\&_{i \in I} \mathcal{X}_{i}$ by $\mathcal{X}^{I}$. In particular, $\mathcal{X}^{\mathbb{N}}$ denotes the product over natural number indexes: it will play a crucial role in the construction of our model of pure $\lambda$-calculus. Given $v \in \mathrm{P}(\mathcal{X}), u \in \mathrm{P}\left(\mathcal{X}^{\mathbb{N}}\right)$, we denote by $v:: u$ the vector in $\mathrm{P}\left(\mathcal{X}^{\mathbb{N}}\right)$ defined by $\pi_{0}(v:: u)=v$ and $\pi_{n+1}(v:: u)=\pi_{n}(u)$.

3) Exponential structure: The functorial promotion ! : $\mathbf{P c o h} \rightarrow$ Pcoh is defined on objects by

$$
\begin{aligned}
|! \mathcal{X}|: & =\mathcal{M}_{\text {fin }}(|\mathcal{X}|), \\
\mathrm{P}(! \mathcal{X}) & :=\left\{x^{!} \text {s.t. } x \in \mathrm{P}(\mathcal{X})\right\}^{\perp \perp},
\end{aligned}
$$

where $x^{!}$is the vector of $\left(\mathbb{R}^{+}\right)^{\mathcal{M}_{\text {fin }}(|\mathcal{X}|)}$ defined by $x_{m}^{!}:=\prod_{a \in \operatorname{Supp}(m)} x_{a}^{m(a)}$, for any $m \in \mathcal{M}_{\text {fin }}(|\mathcal{X}|)$.

The action of ! on a morphism $t \in \operatorname{Pcoh}(\mathcal{X}, \mathcal{Y})$ is defined by, for any $m \in|! \mathcal{X}|, p \in|! \mathcal{Y}|$,

$$
(! t)_{m, p}:=\sum_{r \in L(m, p)}\left[\begin{array}{l}
p \\
r
\end{array}\right]\left(\prod_{(a, b) \in \operatorname{Supp}(r)} t_{a, b}^{r(a, b)}\right)
$$

where $L(m, p)$ is the set of multisets over $|\mathcal{X}| \times|\mathcal{Y}|$ whose first projection is $m$ and whose second projection is $p$ (occurrences do matter) and where

$$
\left[\begin{array}{l}
p \\
r
\end{array}\right]:=\prod_{b \in \operatorname{Supp}(p)} \frac{p(b) !}{\prod_{a \text { s.t. }(a, b) \in \operatorname{Supp}(r)} r(a, b) !}
$$

is the number ${ }^{1}$ of ways of associating the elements of $p$ to the elements of $m$ in order to get $r$. Let us underline that such a coefficient introduces scalars greater than 1. As an example, let $\mathcal{B}:=(1 \& 1)^{\perp}$ and call $\mathrm{t}$, $\mathrm{f}$ the only two elements of its web. Notice that $\mathrm{P}(\mathcal{B})=\left\{x \in\left(\mathbb{R}^{+}\right)^{\{\mathrm{t}, \mathrm{f}\}}\right.$ s.t. $\left.x_{\mathrm{t}}+x_{\mathrm{f}} \leq 1\right\}$. Consider $t \in\left(\mathbb{R}^{+}\right)^{|\mathcal{B}| \times|1|}$ defined by $t_{\mathrm{t}, *}=t_{\mathrm{f}, *}=1$. Notice $t \in \mathbf{P} \operatorname{coh}(\mathcal{B}, 1)$, hence $! t \in \mathbf{P} \operatorname{coh}(! \mathcal{B}, ! 1)$. We have ! $t_{[\mathrm{t}, \mathrm{f}],[*, *]}=$

\footnotetext{
${ }^{1}$ On a side note, note the asymmetry between $p$ and $m$ : if $m=[a, b]$, $p=[c, c]$, then $L(m, p)$ has exactly one element, $[(a, c),(b, c)]$, and the number of ways of getting it is $\left[\begin{array}{c}p \\ {[(a, c),(b, c)]}\end{array}\right]=2$, while, inverting $p$ and $m$, we have that $L(p, m)$ has always one element, $[(c, a),(c, b)]$, but the number of ways of getting it is $\left[\begin{array}{c}m \\ {[(c, a),(c, b)]}\end{array}\right]=1$.
} 
$[*, *]\left[{ }_{0}\right] t_{\mathrm{t}, *} t_{\mathrm{f}, *}=2$. This shows why, in the definition of a PCS, one should take the scalars in $\mathbb{R}^{+}$instead of restricting to $[0,1]$.

Let us underline that adding the coefficient $\left[\begin{array}{l}p \\ r\end{array}\right]$ in the definition of ! $t$ is crucial for ! to be a functor, in fact for having the commutation with the composition (see [DE11, Sect. 1.6]).

The functorial promotion is equipped with a structure of comonad. The counit (also called dereliction) is $d^{\mathcal{X}} \in$ $\operatorname{Pcoh}(! \mathcal{X}, \mathcal{X})$ given by $d_{m, a}^{\mathcal{X}}=\delta_{m,[a]}$. The comultiplication (also called digging) is $p^{\mathcal{X}} \in \mathbf{P} \operatorname{coh}(! \mathcal{X}, ! ! \mathcal{X})$ given by $p_{m, M}^{\mathcal{X}}=$ $\delta_{m, \biguplus M}$, where $\biguplus M$ is the multiset in $|! \mathcal{X}|$ obtained as the multiset union of the multisets in $M \in|! ! \mathcal{X}|$.

4) The coKleisli category Pcoh!: It is induced by the comonad associated with the functorial promotion. The objects of Pcoh! are the PCSs and the set of morphisms $\operatorname{Pcoh}_{!}(\mathcal{X}, \mathcal{Y})$ is equal to $\operatorname{Pcoh}(! \mathcal{X}, \mathcal{Y})$.

The identity $\operatorname{Id}^{\mathcal{X}}$ on $\mathcal{X}$ is the dereliction $d^{\mathcal{X}}$, while the composition $u \circ$ ! $v$ of two morphisms $v \in \operatorname{Pcoh}_{!}(\mathcal{X}, \mathcal{Y})$, $u \in \operatorname{Pcoh}_{!}(\mathcal{Y}, \mathcal{Z})$ is defined by $u \circ ! v:=u \circ ! v \circ p^{\mathcal{X}}$.

As it is known [Gir87], the monoidal closedness of Pcoh is lifted to a cartesian closedness in Pcoh! by Girard's isomorphism between $!(\mathcal{A} \& \mathcal{B})$ and $! \mathcal{A} \otimes ! \mathcal{B}$. The product of a countable family $\left(\mathcal{X}_{i}\right)_{i \in I}$ is the PCS $\&_{i \in I} \mathcal{X}_{i}$ endowed with the projections $\operatorname{Pr}^{j} \in \mathbf{P c o h}_{!}\left(\&_{i \in I} \mathcal{X}_{i}, \mathcal{X}_{j}\right)$ defined by $\operatorname{Pr}^{j}:=\operatorname{pr}^{j} \circ d^{\&_{i \in I} \mathcal{X}_{i}}$. The object of the cokliesli morphisms from $\mathcal{X}$ to $\mathcal{Y}$ is $\mathcal{X} \Rightarrow \mathcal{Y}:=! \mathcal{X} \multimap \mathcal{Y}$, the evaluation $\mathrm{Ev} \in \operatorname{Pcoh}_{!}((\mathcal{X} \Rightarrow \mathcal{Y}) \& \mathcal{X}, \mathcal{Y})$ is defined by $\mathrm{Ev}_{(m, p), a}:=\delta_{m,[(p, a)]}$ and the curryfication of a morphism $v \in \operatorname{Pcoh}_{!}(\mathcal{X} \& \mathcal{Z}, \mathcal{Y})$ is $\operatorname{Cur}(v) \in \operatorname{Pcoh}_{!}(\mathcal{Z}, \mathcal{X} \Rightarrow \mathcal{Y})$ defined by $\operatorname{Cur}(v)_{m,(p, a)}:=v_{(p, m), a}$. Notice that in both Ev and $\operatorname{Cur}(v)$ we are using Girard's isomorphism.

We introduce now entire functions which yield an alternative description of coKleisli morphisms.

Definition 5. A function $f: \mathrm{P}(\mathcal{X}) \rightarrow \mathrm{P}(\mathcal{Y})$ is called entire whenever there exists a matrix $\operatorname{Tr}(f) \in\left(\mathbb{R}^{+}\right)^{\mathcal{M}}$ fin $(|\mathcal{X}|) \times|Y|$ such that $f(x)=\operatorname{Tr}(f) \cdot x^{!}$, for every $x \in \mathrm{P}(\mathcal{X})$.

The name is justified by the analogy with the entire series: the explicit definition of $\operatorname{Tr}(f) \cdot x^{!}$is indeed a power series with coefficients in $\mathbb{R}^{+}$:

$$
\forall b \in|\mathcal{Y}|, f(x)_{b}=\sum_{m \in|! \mathcal{X}|} \operatorname{Tr}(f)_{m, b} \prod_{a \in \operatorname{Supp}(m)} x_{a}^{m(a)} .
$$

The notation $\operatorname{Tr}(f)$ is due to the analogue with the trace of a stable function in Girard's coherence spaces [Gir87]. In fact, entire functions and coKleisli morphisms coincide: on the one hand, we have $\operatorname{Tr}(f) \in \operatorname{Pcoh}(! \mathcal{X} \multimap \mathcal{Y})$ [DE11, Lemma 12], on the other hand, the matrix defining an entire function is unique [DE11, Lemma 19].

Proposition 6. For any $\mathcal{X}, \mathcal{Y}, \operatorname{Tr}$ is a bijection between the entire functions from $\mathrm{P}(\mathcal{X})$ to $\mathrm{P}(\mathcal{Y})$ and the matrices in $\operatorname{Pcoh}(! \mathcal{X} \multimap \mathcal{Y})$. Moreover, $\operatorname{Tr}(g \circ f)=\operatorname{Tr}(g) \circ ! \operatorname{Tr}(f)$ for every $f: \mathrm{P}(\mathcal{X}) \rightarrow \mathrm{P}(\mathcal{Y})$ and $g: \mathrm{P}(\mathcal{Y}) \rightarrow \mathrm{P}(\mathcal{Z})$ entire.

This means that any morphism in $\operatorname{Pcoh}_{!}(\mathcal{X}, \mathcal{Y})$ can be identified with the associated entire function from $\mathrm{P}(\mathcal{X})$ to
$\mathrm{P}(\mathcal{Y})$, and this identification is compatible with composition. Thus, in the sequel we will give a morphism in $\operatorname{Pcoh}_{!}(\mathcal{X}, \mathcal{Y})$ either as a matrix in $\operatorname{Pcoh}(! \mathcal{X} \multimap \mathcal{Y})$ or as a composition of entire maps. As an example, let us describe the evaluation and curryfication, above defined by their matrix, as composition of entire functions. The evaluation morphism $\mathrm{Ev}: \mathrm{P}((\mathcal{X} \Rightarrow \mathcal{Y}) \& \mathcal{X}) \rightarrow \mathrm{P}(\mathcal{Y})$ is the function such that $\operatorname{Ev}(f, x)=f(x)$ for any $(f, x) \in \mathrm{P}(\mathcal{X} \Rightarrow \mathcal{Y}) \times \mathrm{P}(\mathcal{X}) \simeq$ $\mathrm{P}((\mathcal{X} \Rightarrow \mathcal{Y}) \& \mathcal{X})$. Given an entire $f: \mathrm{P}(\mathcal{X} \& \mathcal{Z}) \rightarrow \mathrm{P}(\mathcal{Y})$, its curryfication $\operatorname{Cur}(f): \mathrm{P}(\mathcal{Z}) \rightarrow \mathrm{P}(\mathcal{X} \Rightarrow \mathcal{Y})$ is given by $\operatorname{Cur}(f)(z)(x)=f(x, z)$ for $x \in \mathrm{P}(\mathcal{X}), z \in \mathrm{P}(\mathcal{Z})$.

\section{B. A Reflexive Object in Pcoh}

We describe a reflexive object $\left(\mathcal{D}, \lambda\right.$, app) of $\mathbf{P c o h}_{!}$, giving rise to an extensional model of $\Lambda^{+}$. This means that $\mathcal{D}$ is a PCS, the pair made of $\lambda \in \operatorname{Pcoh}_{!}(\mathcal{D} \Rightarrow \mathcal{D}, \mathcal{D})$ and app $\in$ $\operatorname{Pcoh}_{!}(\mathcal{D}, \mathcal{D} \Rightarrow \mathcal{D})$ is an isomorphism, i.e. app ०! $\lambda=\operatorname{Id}^{\mathcal{D} \Rightarrow \mathcal{D}}$ and $\lambda \circ$ ! app $=\operatorname{Id}^{\mathcal{D}}$.

1) The object $\mathcal{D}$ : The PCS $\mathcal{D}$ is defined in Figure 3. It is obtained by iterating the operation $\mathcal{X} \mapsto\left(! \mathcal{X}^{\mathbb{N}}\right)^{\perp}$ starting from the empty-web PCS $\mathcal{D}_{0}:=(\emptyset, 0)$. More precisely, notice $\left|\mathcal{D}_{\ell+1}\right|=\left|\left(! \mathcal{D}_{\ell}^{\mathbb{N}}\right)\right|$. So $\left\langle v, u^{!}\right\rangle$is well-defined for any $v \in$ $\left(\mathbb{R}^{+}\right)^{\left|\mathcal{D}^{\ell+1}\right|}$ and $u \in \mathrm{P}\left(\mathcal{D}_{\ell}^{\mathbb{N}}\right)$, as the vector $u^{!}$is in $\mathrm{P}\left(! \mathcal{D}_{\ell}^{\mathbb{N}}\right)$. Besides, by definition $\mathrm{P}\left(\mathcal{D}_{\ell+1}\right)=\left\{u^{!} \text {s.t. } u \in \mathrm{P}\left(\mathcal{D}_{\ell}^{\mathbb{N}}\right)\right\}^{\perp}=$ $\left\{u^{!} \text {s.t. } u \in \mathrm{P}\left(\mathcal{D}_{\ell}^{\mathbb{N}}\right)\right\}^{\perp \perp \perp}=\mathrm{P}\left(\left(! \mathcal{D}_{\ell}^{\mathbb{N}}\right)^{\perp}\right)$. So we get that $\mathcal{D}_{\ell+1}=\left(! \mathcal{D}_{\ell}^{\mathbb{N}}\right)^{\perp}$, hence $\mathcal{D}_{\ell+1}$ is a PCS.

In [DE11, Sect. 2], $\mathcal{D}$ is shown to be the lub of $\left\{\mathcal{D}_{\ell}\right\}_{\ell \in \mathbb{N}}$, an increasing chain with respect to the following order:

$$
\mathcal{X} \subseteq \mathcal{Y} \Leftrightarrow\left\{\begin{array}{l}
|\mathcal{X}| \subseteq|\mathcal{Y}| \\
\mathrm{P}(\mathcal{X})=\left\{\left.v\right|_{|\mathcal{X}|} \text { s.t. } v \in \mathrm{P}(\mathcal{Y})\right\}
\end{array}\right.
$$

Moreover, the operation $\mathcal{X} \mapsto\left(! \mathcal{X}^{\mathbb{N}}\right)^{\perp}$ is Scott continuous (i.e. monotone and preserving directed lub), hence $\mathcal{D}$ is its least fixed point by Kleene-Tarski Theorem [Tar55].

2) The isomorphism pair $\lambda$ and app: Let us introduce a convenient notation. Let $m \in\left|! \mathcal{D}_{\ell}\right|=\mathcal{M}_{\text {fin }}\left(\left|\mathcal{D}_{\ell}\right|\right)$ and $d \in$ $\left|\mathcal{D}_{\ell+1}\right|=\mathcal{M}_{\text {fin }}\left(\cup_{n \in \mathbb{N}}\{n\} \times\left|\mathcal{D}_{\ell}\right|\right)$, we set:

$$
m:: d:=[(0, c) \text { s.t. } c \in m] \uplus[(n+1, c) \text { s.t. }(n, c) \in d]
$$

Notice that $m:: d \in \mathcal{D}_{\ell+1}$. Conversely, for any point in $d \in \mathcal{D}_{\ell+1}$ there is a unique $m \in\left|! \mathcal{D}_{\ell}\right|$, potentially empty, and $d^{\prime} \in\left|\mathcal{D}_{\ell+1}\right|$ such that $d=m:: d^{\prime}$.

The empty multiset is a remarkable element of $|\mathcal{D}|$ that we denote $\star$. In particular, we have $\star=[]:: \star$. This notation underlines an isomorphism ${ }^{2}$ between the webs of $\mathcal{D}$ and $\mathcal{D} \Rightarrow$ $\mathcal{D}$, that is $\mathcal{M}_{\text {fin }}(|\mathcal{D}|) \times|\mathcal{D}|$.

\footnotetext{
${ }^{2}$ As described in [BEM], Equation (5) defines an isomorphism between $\mathcal{M}_{\text {fin }}\left(\cup_{n \in \mathbb{N}}\{n\} \times\left|\mathcal{D}_{\ell}\right|\right)$ and the set $\mathcal{M}_{\text {fin }}\left(\left|\mathcal{D}_{\ell}\right|\right)^{(\omega)}$ of the infinite sequences of finite multisets of $\left|\mathcal{D}_{\ell}\right|$ almost everywhere empty. In fact, for every $d \in$ $\mathcal{D}_{\ell+1}$ there are a unique number $k$ and multisets $m_{0}, \ldots, m_{k-1} \in\left|! \mathcal{D}_{\ell}\right|$ such that $m_{k-1} \neq[]$, and $d=m_{0}:: \ldots m_{k}::$ []. Then, $d$ is associated with the sequence having the first $k$ elements respectively equal to $m_{0}, \ldots, m_{k-1}$ and all the other elements equal to the empty multiset. This isomorphism justifies the notation of $m:: d$ as an append operation and extends into an isomorphism between $\mathcal{M}_{\text {fin }}\left(\cup_{n \in \mathbb{N}}\{n\} \times|\mathcal{D}|\right)$ and $\mathcal{M}_{\text {fin }}(|\mathcal{D}|)^{(\omega)}$.
} 


$$
\begin{aligned}
& \left|\mathcal{D}_{0}\right|:=\emptyset \\
& \left|\mathcal{D}_{\ell+1}\right|:=\mathcal{M}_{\text {fin }}\left(\cup_{n \in \mathbb{N}}\{n\} \times\left|\mathcal{D}_{\ell}\right|\right) \\
& \mathrm{P}\left(\mathcal{D}_{0}\right):=\mathbf{0} \\
& |\mathcal{D}|:=\bigcup_{\ell \in \mathbb{N}}\left|\mathcal{D}_{\ell}\right| \\
& \begin{aligned}
\mathrm{P}\left(\mathcal{D}_{\ell+1}\right): & =\{ \\
\mathrm{P}(\mathcal{D}) & :=\{
\end{aligned} \\
& \left.v \in\left(\mathbb{R}^{+}\right)^{\left|\mathcal{D}_{\ell+1}\right|} \text { s.t. } \forall u \in \mathrm{P}\left(\mathcal{D}_{\ell}^{\mathbb{N}}\right),\left\langle v, u^{!}\right\rangle \leq 1\right\} \\
& \left.v \in\left(\mathbb{R}^{+}\right)^{|\mathcal{D}|} \text { s.t. } \forall \ell \in \mathbb{N}, \forall u \in \mathrm{P}\left(\mathcal{D}_{\ell}^{\mathbb{N}}\right),\left\langle\left. v\right|_{\left|\mathcal{D}_{\ell+1}\right|}, u^{!}\right\rangle \leq 1\right\}
\end{aligned}
$$

Fig. 3. Definition of $\mathcal{D}$ as the least fixpoint of the operation $\mathcal{X} \mapsto\left(! \mathcal{X}^{\mathbb{N}}\right)^{\perp}$. Recall the notation $\left.v\right|_{\left|\mathcal{D}_{\ell+1}\right|} \in\left(\mathbb{R}^{+}\right)^{\left|\mathcal{D}_{\ell+1}\right|}$ obtained by restricting $v \in\left(\mathbb{R}^{+}\right)^{|\mathcal{D}|}$ to the indexes $\left|\mathcal{D}_{\ell+1}\right| \subseteq|\mathcal{D}|$.

We set $\lambda \in \mathbf{P c o h}_{!}(\mathcal{D} \Rightarrow \mathcal{D}, \mathcal{D})$ and app $\in \mathbf{P c o h}_{!}(\mathcal{D}, \mathcal{D} \Rightarrow$ $\mathcal{D})$ as follows: for any $p \in \mathcal{M}_{\text {fin }}(|\mathcal{D} \Rightarrow \mathcal{D}|), m, q \in$ $\mathcal{M}_{\text {fin }}(|\mathcal{D}|)$, and $d \in|\mathcal{D}|$,

$$
\lambda_{p, m:: c}:=\delta_{p,[(m, d)]}, \quad \operatorname{app}_{q,(m, d)}:=\delta_{q,[m:: d]} .
$$

An easy computation shows that app $\circ ! \lambda=\operatorname{Id}^{\mathcal{D} \Rightarrow \mathcal{D}}$ and $\lambda \circ$ ! app $=\mathrm{Id}^{\mathcal{D}}$, so that $(\mathcal{D}, \lambda$, app $)$ yields an extensional model of pure $\lambda$-calculus.

3) Properties of $(\mathcal{D}, \lambda$, app): It is significant that $\mathcal{D}$ satisfies two different recursive equations: $\mathcal{X}=\left(! \mathcal{X}^{\mathbb{N}}\right)^{\perp}$ and $\mathcal{X}=\mathcal{X} \Rightarrow$ $\mathcal{X}$. The first gives the construction of $\mathcal{D}$ and, in fact, $\mathcal{D}$ is its minimal solution (with respect to $\subseteq$ ). The second equation is needed to interpret the pure $\lambda$-calculus, however $\mathcal{D}$ is not its minimal solution, since the empty-web PCS $\mathcal{D}_{0}$ trivially satisfies $\mathcal{D}_{0}=\mathcal{D}_{0} \Rightarrow \mathcal{D}_{0}$.

Remark that $\mathcal{D}$ is isomorphic to $\mathcal{D}^{\mathbb{N}} \Rightarrow \perp$. In fact, if $\mathrm{P}(\mathcal{D})$ is meant to contain the denotations of terms, the vectors in $\mathrm{P}\left(\mathcal{D}^{\mathbb{N}}\right)$ morally express infinite stacks of terms, whose promotion play the role of the environments.

Our main result will give a computational meaning to the values of the vectors in $\mathrm{P}(\mathcal{D})$ associated with the indexes in $\left|\mathcal{D}_{2}\right|$. By means of the notations introduced in Subsection III-B2, we have $\left|\mathcal{D}_{1}\right|=\{\star\}$ and

$$
\left|\mathcal{D}_{2}\right|=\left\{\begin{array}{r}
m_{0}:: \cdots:: m_{k}:: \star \text { s.t. } k \in \mathbb{N}, \forall i \leq k \\
m_{i}=[\star, \ldots, \star] \in \mathcal{M}_{\text {fin }}\left(\left|\mathcal{D}_{1}\right|\right)
\end{array}\right\} .
$$

\section{Interpretation of the Terms of $\Lambda^{+}$}

The closed terms of $\Lambda^{+}$are interpreted as vectors in $\mathrm{P}(\mathcal{D})$. In the general case, given a term $M$ and a list $\Gamma$ of pairwise different variables containing all the free variables of $M$, the interpretation of $M$ is a morphism $\llbracket M \rrbracket^{\Gamma} \in \mathbf{P c o h}_{!}\left(\mathcal{D}^{\Gamma}, \mathcal{D}\right)$, which can be seen as an entire function:

$$
\llbracket M \rrbracket^{\Gamma}: \mathrm{P}\left(\mathcal{D}^{\Gamma}\right) \rightarrow \mathrm{P}(\mathcal{D}) \text {. }
$$

The definition of $\llbracket M \rrbracket^{\Gamma}$ is given in Figure 4 , by structural induction on $M$. Using the notation of that figure, we recall that $\pi_{x}(v) \in \mathrm{P}(\mathcal{D})$ is the $x$-th component of $v \in \mathrm{P}\left(\mathcal{D}^{\Gamma}\right)$, for $x \in \Gamma$. Also, recall the writing $u:: v$ denotes the vector in $\mathrm{P}\left(\mathcal{D}^{x, \Gamma}\right)$ whose $x$-th component is $u \in \mathrm{P}(\mathcal{D})$ and whose components in $\Gamma$ are given by $v \in \mathrm{P}\left(\mathcal{D}^{\Gamma}\right)$. Finally, $u \mapsto$ $\llbracket M \rrbracket^{x, \Gamma}(u:: v)$ denotes the entire function mapping any $u \in$ $\mathrm{P}(\mathcal{D})$ to $\llbracket M \rrbracket^{x, \Gamma}(u:: v) \in \mathrm{P}(\mathcal{D})$. We will simply write $\llbracket M \rrbracket$ in case $M$ is a closed term.

Notice that $*$ is interpreted by the basis vector $e_{\star}$ in the direction of the empty multiset $\star \in|\mathcal{D}|$, and $+_{p}$ is interpreted

$$
\begin{aligned}
\llbracket * \rrbracket^{\Gamma}(v) & :=e_{\star} \\
\llbracket x \rrbracket^{\Gamma}(v) & :=\pi_{x}(v) \\
\llbracket \lambda x . M \rrbracket^{\Gamma}(v) & :=\lambda\left(u \mapsto \llbracket M \rrbracket^{x, \Gamma}(u:: v)\right) \\
\llbracket M N \rrbracket^{\Gamma}(v) & :=\operatorname{app}\left(\llbracket M \rrbracket^{\Gamma}(v)\right)\left(\llbracket N \rrbracket^{\Gamma}(v)\right) \\
\llbracket M+{ }_{p} N \rrbracket^{\Gamma}(v) & :=p \llbracket M \rrbracket^{\Gamma}(v)+(1-p) \llbracket N \rrbracket^{\Gamma}(v)
\end{aligned}
$$

Fig. 4. Interpretation of a term in $\Lambda^{+}$as an entire function from $\mathrm{P}\left(\mathcal{D}^{\Gamma}\right)$ to $\mathrm{P}(\mathcal{D})$.

by the $p$-weighted sum. Apart from these, the interpretation follows the one determined by the categorical model of the pure $\lambda$-calculus given by the cartesian closed structure of the category $\mathbf{P c o h} !$ and the reflexive object $(\mathcal{D}, \lambda$, app). More precisely, $\llbracket x \rrbracket^{\Gamma}$ is the $x$-th projection of the product $\mathcal{D}^{\Gamma}$, $\llbracket \lambda x \cdot M \rrbracket^{\Gamma}(v)=\lambda \circ$ ! Cur $\left(\llbracket M \rrbracket^{x, \Gamma}\right) \circ$ ! $v$ and $\llbracket M N \rrbracket^{\Gamma}(v)=$ Ev o! (app o! $\left.\left(\llbracket M \rrbracket^{\Gamma} \circ ! v\right), \llbracket N \rrbracket^{\Gamma} \circ ! v\right)$.

Proposition 7 (Invariance of the intepretation). For every term $M \in \Lambda^{+}$, and sequence $\Gamma \supseteq \mathrm{FV}(M)$, we have:

$$
\llbracket M \rrbracket^{\Gamma}=\sum_{N \in \Lambda^{+}} \operatorname{Red}_{M, N} \llbracket N \rrbracket^{\Gamma} .
$$

Proof: It is a standard structural induction on $M$. The case $M=(\lambda x . N) L$ is achieved by means of the substitution lemma $\llbracket N[L / x] \rrbracket^{\Gamma}(v)=\llbracket N \rrbracket^{x, \Gamma}\left(\llbracket L \rrbracket^{\Gamma}(v), v\right)$, inferred by a straightforward structural induction.

\section{The ADEQUACY OF $\mathcal{D}$ FOR $\Lambda^{+}$}

Our goal is to achieve Theorem 22: the probability that a closed term $M$ reaches a head normal form is equal to the sum of the values of $\llbracket M \rrbracket$ on the points of $\left|\mathcal{D}_{2}\right|$. First, we prove $\sum_{d \in\left|\mathcal{D}_{2}\right|} \llbracket M \rrbracket_{d} \geq \sum_{H \in \mathrm{hnf}} \operatorname{Red}_{M, H}^{\infty}$ (Proposition 11). This result is an easy consequence of the invariance of the interpretation under head-reduction (Proposition 7) and of the fact that $\sum_{d \in\left|\mathcal{D}_{2}\right|} \llbracket M \rrbracket_{d}=1$ whenever $M$ is a head normal form (Lemma 10). Then, we turn to the converse inequality, $\sum_{d \in\left|\mathcal{D}_{2}\right|} \llbracket M \rrbracket_{d} \leq \sum_{H \in \mathrm{hnf}} \operatorname{Red}_{M, H}^{\infty}$. Its proof is by far more delicate. In fact it corresponds to a quantitative version of the sensibility of Scott's model with respect to the standard $\lambda$-calculus [Hyl76]: a $\lambda$-term with no head normal form is interpreted by the bottom element of the model. The inequality will be proved in Section IV-A, using a notion of formal approximation relating the syntactical behavior of the closed terms in $\Lambda^{+}$with their denotations in $\mathcal{D}$. 
The next two lemmas are preliminary to the whole section. Lemma 8 precises the intuition about pairing and orthogonal we gave in the introduction to probabilistic coherence spaces (Section III). Recall that the interpretration of a term $\mathrm{M}$ is a vector $\llbracket M \rrbracket$ in $\mathrm{P}(\mathcal{D})$ and the interpretration of an environment intended as an infinite stack of terms is a vector $u$ in $\mathrm{P}\left(\mathcal{D}^{\mathbb{N}}\right)$. Then, the interaction of a term and an environment is given by pairing and promotion: $\left\langle\llbracket M \rrbracket, u^{!}\right\rangle$. We recall the notation of Section III-A2: if $r \in \mathrm{P}(\mathcal{D})$ and $u \in \mathrm{P}\left(\mathcal{D}^{\mathbb{N}}\right), r:: u$ is the vector in $\mathrm{P}\left(\mathcal{D}^{\mathbb{N}}\right)$ defined by $\pi_{0}(r:: u)=r$ and $\pi_{n+1}(r:: u)=\pi_{n}(u)$.

Lemma 8. For every $v, r \in \mathrm{P}(\mathcal{D})$ and $u \in \mathrm{P}\left(\mathcal{D}^{\mathbb{N}}\right)$,

$$
\left\langle\operatorname{app}(v)(r), u^{!}\right\rangle=\left\langle v,(r:: u)^{!}\right\rangle \text {. }
$$

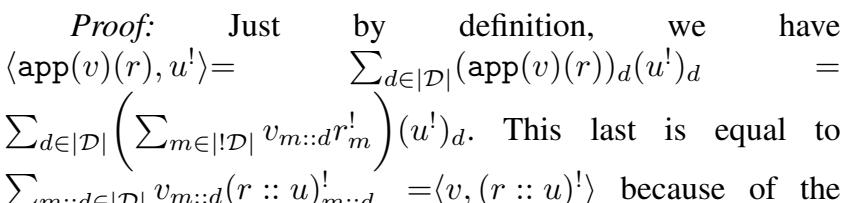
$\sum_{m:: d \in|\mathcal{D}|} v_{m:: d}(r:: u)_{m:: d}^{!}=\langle v,(r:: u)$
isomorphism between $|\mathcal{D} \Rightarrow \mathcal{D}|$ and $|\mathcal{D}|$

Lemma 9 states that $\sum_{d \in\left|\mathcal{D}_{2}\right|} \llbracket M \rrbracket_{d}$ estimates the behavior of $M$ when applied to an infinite stack made of $*$ terms. We will denote $e_{\vec{\star}}$ the vector of $\mathrm{P}\left(\mathcal{D}^{\mathbb{N}}\right)$ that embodied this stack. It is defined by, for every $i \in \mathbb{N}, \pi_{i}\left(e_{\vec{\star}}\right)=e_{\star} \in \mathrm{P}(\mathcal{D})$. Notice $e_{\vec{\star}}=e_{\star}:: e_{\vec{\star}}$.

Lemma 9. The vector $\left(e_{\vec{*}}\right)^{!}$is in $\mathrm{P}\left(\mathcal{D}^{\perp}\right)$. Besides, for any $v \in \mathrm{P}(\mathcal{D}), \sum_{d \in\left|\mathcal{D}_{2}\right|} v_{d}=\left\langle v,\left(e_{\vec{k}}\right)^{!}\right\rangle \leq 1$.

Proof: Since $e_{\vec{\star}} \in \mathrm{P}\left(\mathcal{D}^{\mathbb{N}}\right),\left(e_{\vec{\star}}\right)^{!} \in \mathrm{P}\left(!\left(\mathcal{D}^{\mathbb{N}}\right)\right)=\mathrm{P}\left(\mathcal{D}^{\perp}\right)$.

From the description of $\left|\mathcal{D}_{2}\right|$ (Subsection III-B3), we get $\left(e_{\vec{\star}}\right)_{d}^{!}=1$ if $d \in\left|\mathcal{D}_{2}\right|$, otherwise it is equal to 0 . Then we have, $\sum_{d \in\left|\mathcal{D}_{2}\right|} v_{d}=\sum_{d \in|\mathcal{D}|} v_{d}\left(e_{\vec{\star}}\right)_{d}^{!}=\left\langle v,\left(e_{\vec{\star}}\right)^{!}\right\rangle \leq 1$, since $v \in \mathrm{P}(\mathcal{D})$ and $\left(e_{\vec{*}}\right)^{!} \in \mathrm{P}\left(\mathcal{D}^{\perp}\right)$.

Lemma 10. Let $M \in \Lambda_{0}^{+}$. If $M$ is a head normal form, then $\sum_{d \in\left|\mathcal{D}_{2}\right|} \llbracket M \rrbracket_{d}=1$.

Proof: By Lemma 9, the claim is equivalent to $\left\langle\llbracket M \rrbracket,\left(e_{\vec{\star}}\right)^{!}\right\rangle=1$, for a closed head normal form $M$. By definition, $M$ is of the shape $\lambda x_{1} \ldots \lambda x_{\ell} . H M_{1} \ldots M_{m}$, where $H$ is either $*$ or a variable in $\left\{x_{1}, \ldots, x_{\ell}\right\}$. Since $e_{\vec{\star}}=e_{\star}:: e_{\vec{\star}}$, we can apply the equation of Lemma 8 from right-to-left, using the interpretation of Figure 4 and the retraction property app $\circ_{!} \lambda=\mathrm{Id}$, we have $\left\langle\llbracket M \rrbracket,\left(e_{\vec{\star}}\right)^{!}\right\rangle=\left\langle\llbracket H M_{1} \ldots M_{m} \rrbracket^{\Gamma}\left(e_{\star}^{\ell}\right),\left(e_{\vec{\star}}\right)^{!}\right\rangle$, where $\Gamma=\left(x_{1}, \ldots, x_{\ell}\right)$ and $e_{\star}^{\ell}$ denotes the sequence of $\ell$ vectors $e_{\star}$. Then, by unfolding the interpretation of applications, we get $\llbracket H M_{1} \ldots M_{m} \rrbracket^{\Gamma}\left(e_{\star}^{\ell}\right)=$ $\operatorname{app}\left(\ldots \operatorname{app}\left(e_{\star}\right)\left(\llbracket M_{1} \rrbracket^{\Gamma}\left(e_{\star}^{\ell}\right)\right) \ldots\right)^{1}\left(\llbracket M_{m} \rrbracket^{\Gamma}\left(e_{\star}^{\ell}\right)\right) . \quad$ Finally, a computation gives $\operatorname{app}\left(e_{\star}\right)(u)=e_{\star}$ for any $u \in \mathcal{D}$, hence $\left\langle\llbracket M \rrbracket,\left(e_{\vec{\star}}\right)^{!}\right\rangle=\left\langle e_{\star},\left(e_{\vec{\star}}\right)^{!}\right\rangle=1$.

Proposition 11. Let $M \in \Lambda_{0}^{+}$, we have $\sum_{d \in\left|\mathcal{D}_{2}\right|} \llbracket M \rrbracket_{d} \geq$ $\sum_{H \in \text { hnf }} \operatorname{Red}_{M, H}^{\infty}$.

Proof: The proof is made in two steps: first, we prove by induction on $n$ that $\sum_{d \in\left|\mathcal{D}_{2}\right|} \llbracket M \rrbracket_{d} \geq \sum_{H \in \mathrm{hnf}} \operatorname{Red}_{M, H}^{n}$, then, the result follows from $\sum_{H \in \mathrm{hnf}} \operatorname{Red}_{M, H}^{\infty}=$ $\sum_{H \in \mathrm{hnf}}\left(\sup _{n=0}^{\infty} \operatorname{Red}_{M, H}^{n}\right)=\sup _{n=0}^{\infty}\left(\sum_{H \in \mathrm{hnf}} \operatorname{Red}_{M, H}^{n}\right)$ that holds since $\operatorname{Red}_{M, H}^{n}$ is an increasing positive sequence by Proposition 1 .

For the base case, recall that $\sum_{H \in \text { hnf }} \operatorname{Red}_{M, H}^{n} \leq 1$ always holds, since $\operatorname{Red}^{n}$ is a stochastic matrix for every $n \in \mathbb{N}$. Now, $\sum_{H \in \text { hnf }} \operatorname{Red}_{M, H}^{0}$ is different from zero only when $M$ is in head normal form. But then, thanks to Lemma 10, $\sum_{d \in\left|\mathcal{D}_{2}\right|} \llbracket M \rrbracket_{d}=1$ and we are done.

For the induction step, Proposition 7 restricted to $\left|\mathcal{D}_{2}\right|$ yields to $\sum_{d \in\left|\mathcal{D}_{2}\right|} \llbracket M \rrbracket_{d}=\sum_{N \in \Lambda_{0}^{+}} \operatorname{Red}_{M, N}\left(\sum_{d \in\left|\mathcal{D}_{2}\right|} \llbracket N \rrbracket_{d}\right)$. By induction hypothesis, $\sum_{d \in\left|\mathcal{D}_{2}\right|} \llbracket N \rrbracket_{d} \geq \sum_{H \in \operatorname{hnf}} \operatorname{Red}_{N, H}^{n}$ for every $N \in \Lambda_{0}^{+}$. Hence $\sum_{d \in\left|\mathcal{D}_{2}\right|} \llbracket M \rrbracket_{d} \geq$ $\sum_{H \in \text { hnf }} \sum_{N \in \Lambda_{0}^{+}} \operatorname{Red}_{M, N} \operatorname{Red}_{N, H}^{n}$, and we conclude since by definition $\operatorname{Red}_{M, H}^{n+1}:=\sum_{N \in \Lambda^{+}} \operatorname{Red}_{M, N} \operatorname{Red}_{N, H}^{n}$.

\section{A. Formal Approximation}

The goal is to prove the converse of Proposition 11. This follows easily from Lemma 21 . We adapt the technique of logical relations (see e.g. [Plo77], [Rey]) to our quantitive framework. The idea is to find a relation $\triangleleft$ between vectors and terms relating the values of the firsts to the computational behavior of the seconds. Basically, one extends the operation on PCSs defining $\mathcal{D}$ to an operation $\Phi$ acting on the relations in $\mathrm{P}(\mathcal{D}) \times \Lambda_{0}^{+}$(Definition 12). Then, $\triangleleft$ is the result of the closure by $\Phi$ of the relation between $v$ and $M$ defined by $\sum_{d \in\left|\mathcal{D}_{2}\right|} v_{d} \leq \sum_{H \in \text { hnf }} \operatorname{Red}_{M, H}^{\infty}$. However, the operation $\Phi$ is not monotonic, hence finding its closure is not trivial. We then use a technique due to Pitts [Pit], consisting in deriving $\triangleleft$ from a fixed point of a monotonic operation $\Psi$ (Definition 13, Proposition 16) associated with $\Phi$.

Definition 12. For any relation $R \subseteq \mathrm{P}(\mathcal{D}) \times \Lambda_{0}^{+}$, we define the relation $\Phi(R) \subseteq \mathrm{P}(\mathcal{D}) \times \Lambda_{0}^{+}$as follows:

$$
\left\{\begin{array}{cl}
(v, M) \text { s.t. } & \forall u \in \mathrm{P}\left(\mathcal{D}^{\mathbb{N}}\right), \forall n \in \mathbb{N}, \\
& \forall N_{0}, \ldots, N_{n-1} \in \Lambda_{0}^{+} \\
& \text {if } \forall i<n,\left(\pi_{i}(u), N_{i}\right) \in R \text { and } \\
& \text { if } \forall i \geq n, \pi_{i}(u)=e_{\star}, \text { then } \\
& \left\langle v, u^{!}\right\rangle \leq \sum_{H \in \text { hnf }} \operatorname{Red}_{\left(M N_{0} \ldots N_{n-1}\right), H}^{\infty}
\end{array}\right\}
$$

Let us underline the analogy ${ }^{3}$ between $\Phi$ and the operation $\mathcal{X} \mapsto\left(! \mathcal{X}^{\mathbb{N}}\right)^{\perp}$ defining our reflexive object $\mathcal{D}$. Indeed, $\mathrm{P}\left(\left(! \mathcal{X}^{\mathbb{N}}\right)^{\perp}\right)$ is the set of those vectors $v \in\left(\mathbb{R}^{+}\right)\left|! \mathcal{X}^{\mathbb{N}}\right|$ such that for all $u \in\left(\mathbb{R}^{+}\right)^{\left|\mathcal{X}^{\mathbb{N}}\right|}$, if for every $i \in \mathbb{N}, \pi_{i}(u) \in \mathrm{P}(\mathcal{X})$, then $\left\langle v, u^{!}\right\rangle \leq 1$.

By $e_{\vec{\star}} \in \mathrm{P}\left(\mathcal{D}^{\mathbb{N}}\right)$ and Lemma $9,(v, M) \in \Phi(R)$ entails $\sum_{d \in\left|\mathcal{D}_{2}\right|} v_{d}\left\langle v,\left(e_{\vec{\star}}\right)^{!}\right\rangle \leq \sum_{H \in \mathrm{hnf}} \operatorname{Red}_{M, H}^{\infty}$. Indeed, we will

\footnotetext{
${ }^{3}$ The only slight difference between the action of $\Phi$ and that of $\mathcal{X} \mapsto$ $\left(! \mathcal{X}^{\mathbb{N}}\right)^{\perp}$ is that the former asks to test the pairs $\left(\pi_{i}(u), N_{i}\right)$ only for a finite set of indexes, then requiring $\pi_{i}(u)=e_{\star}$. This difference is a technical convenience: one could define $\Phi$ by testing an infinite family of pairs $\left(\pi_{i}(u), N_{i}\right)$ and then studying a kind of abstract machine implementing the head-reduction of the application of $M$ to the infinite stack of terms $N_{0}, N_{1}, \ldots$ Then, it turns out that $\sum_{H \in \mathrm{hnf}} \operatorname{Red}_{\left(M N_{0} \ldots N_{n-1}\right), H}^{\infty}$ is equal to the probability of the termination of such a machine when run on the stack $N_{0}, \ldots, N_{n-1}, *, *, *, \ldots$.
} 
define a relation $\triangleleft$ such that $(\llbracket M \rrbracket, M) \in \Phi(R)$ for any $M \in \Lambda_{0}^{+}$. The relation $\triangleleft$ is in fact a fixed point for $\Phi$.

Definition 13. Given a pair $\left(R^{+}, R^{-}\right) \in\left(\mathcal{P}\left(\mathcal{D} \times \Lambda_{0}^{+}\right)\right)^{2}$, let $\Psi\left(R^{+}, R^{-}\right):=\left(\Phi\left(R^{-}\right), \Phi\left(R^{+}\right)\right) \in\left(\mathcal{P}\left(\mathcal{D} \times \Lambda_{0}^{+}\right)\right)^{2}$. The order $\sqsubseteq$ on $\left(\mathcal{P}\left(\mathcal{D} \times \Lambda_{0}^{+}\right)\right)^{2}$ is $\left(R_{1}, R_{2}\right) \sqsubseteq\left(R_{3}, R_{4}\right)$ iff $R_{1} \subseteq R_{3}$ and $R_{2} \supseteq R_{4}$. Clearly, $\sqsubseteq$ defines a complete lattice on $\left(\mathcal{P}\left(\mathcal{D} \times \Lambda_{0}^{+}\right)\right)^{2}$. We denote by $\Pi$ its glb and we consider the glb of the set of the pre-fixed points of $\Psi$ : $\left(\triangleleft^{+}, \triangleleft^{-}\right):=\prod\left\{\left(R^{+}, R^{-}\right)\right.$s.t. $\left.\Psi\left(R^{+}, R^{-}\right) \sqsubseteq\left(R^{+}, R^{-}\right)\right\}$

Lemma 14. We have $\Psi\left(\triangleleft^{+}, \triangleleft^{-}\right)=\left(\triangleleft^{+}, \triangleleft^{-}\right)$. In particular, $\triangleleft^{+}=\Phi\left(\triangleleft^{-}\right)$and $\triangleleft^{-}=\Phi\left(\triangleleft^{+}\right)$.

Proof: As $R \subseteq R^{\prime}$ entails $\Phi\left(R^{\prime}\right) \subseteq \Phi(R), \Psi$ is monotone increasing with respect to $\sqsubseteq$. Hence, by Tarski's Theorem on fixed points [Tar55] the glb of the set of the pre-fixed points of $\Psi$ is the least fixed point of $\Psi$. In particular, by definition of $\Psi, \Phi\left(\triangleleft^{+}\right)=\triangleleft^{-}$and $\Phi\left(\triangleleft^{-}\right)=\triangleleft^{+}$.

Proposition 16 shows that actually $\triangleleft^{+}=\triangleleft^{-}$, so this is a fixed point for $\Phi$. The inclusion $\triangleleft^{+} \subseteq \triangleleft^{-}$follows easily from the previous lemma, while the proof of the converse $\triangleleft^{-} \subseteq \triangleleft^{+}$ uses the approximations of the vectors in $\mathrm{P}(\mathcal{D})$ given by the chain $\left\{\mathcal{D}_{\ell}\right\}_{\ell \in \mathbb{N}}$ of which $\mathcal{D}$ is the limit. The following lemma is needed in the proof of Proposition 16.

Lemma 15. For any relation $R \subseteq \mathrm{P}(\mathcal{D}) \times \Lambda_{0}^{+}$and any term $M \in \Lambda_{0}^{+}$, the set $\Phi(R)_{M}:=\{v$ s.t. $(v, M) \in \Phi(R)\}$ contains $\mathbf{0}$, is downward and chain closed, i.e. if $v^{\prime} \leq v \in \Phi(R)_{M}$, then $v^{\prime} \in \Phi(R)_{M}$ and if $\left(v_{j}\right)_{j \in \mathbb{N}} \subseteq \phi(R)_{M}$ is an increasing family, then $\sup _{j \in \mathbb{N}} v_{j} \in \phi(R)_{M}$.

Proof: First, $\mathbf{0} \in \Phi(R)_{M}$ : by the linearity of the pairing, $\left\langle\mathbf{0}, u^{!}\right\rangle=0 \leq \sum_{H \in \operatorname{hnf}} \operatorname{Red}_{\left(M N_{0} \ldots N_{n-1}\right), H}^{\infty}$. Then, $(v, M) \in$ $\Phi(R)$ gives $\left(v^{\prime}, M\right) \in \Phi(R)$ : indeed, being coefficients positive reals, for any $v^{\prime} \leq v,\left\langle v^{\prime}, u^{!}\right\rangle \leq\left\langle v, u^{!}\right\rangle$.

As for the sup-closedness, suppose $\left(v_{j}\right)_{j \in \mathbb{N}} \subseteq \Phi(R)_{M}$ is increasing, then, by Proposition 4, we have $\sup _{j \in \mathbb{N}} v_{j} \in$ $\mathrm{P}(\mathcal{D})$. By Definition 12, we must also prove that $\forall u \in$ $\mathrm{P}\left(\mathcal{D}^{\mathbb{N}}\right), \forall n \in \mathbb{N}, \forall N_{0}, \ldots, N_{n-1}$, such that $\forall i<n$, $\left(\pi_{i}(u), N_{i}\right) \in R$ and $\forall i \geq n, \pi_{i}(u)=e_{\star}$, we have $\left\langle\sup _{j \in \mathbb{N}} v_{j}, u^{!}\right\rangle \leq \sum_{H \in \text { hnf }} \operatorname{Red}_{\left(M N_{0} \ldots N_{n-1}\right), H}^{\infty}$. By hypothesis, $\left\langle v_{j}, u^{!}\right\rangle \leq \sum_{H \in \operatorname{hnf}} \operatorname{Red}_{\left(M N_{0} \ldots N_{n-1}\right), H}^{\infty}$, for every $j$. Since $\left(v_{j}\right)_{j \in \mathbb{N}}$ is increasing and all coefficients are positive reals, its lub is also its limit, hence we get $\left\langle\sup _{j \in \mathbb{N}} v_{j}, u^{!}\right\rangle=$ $\sup _{j \in \mathbb{N}}\left\langle v_{j}, u^{!}\right\rangle \leq \sum_{H \in \operatorname{hnf}} \operatorname{Red}_{\left(M N_{0} \ldots N_{n-1}\right), H}^{\infty}$.

Proposition 16. We have $\triangleleft^{+}=\triangleleft^{-}$, which is then a fixed point of $\Phi$. From now on, we denote it simply by $\triangleleft$.

Proof: We prove $\triangleleft^{+} \subseteq \triangleleft^{-}$. Remark that $\left(\triangleleft^{-}, \triangleleft^{+}\right)$is a pre-fixed point of $\Psi$, i.e. $\Psi\left(\triangleleft^{-}, \triangleleft^{+}\right) \sqsubseteq\left(\triangleleft^{-}, \triangleleft^{+}\right)$: indeed, this is equivalent to $\Phi\left(\triangleleft^{+}\right) \subseteq \triangleleft^{-}$and $\Phi\left(\triangleleft^{-}\right) \supseteq \triangleleft^{+}$, which holds by Lemma 14. Then we have $\left(\triangleleft^{+}, \triangleleft^{-}\right) \sqsubseteq\left(\triangleleft^{-}, \triangleleft^{+}\right)$ since $\left(\triangleleft^{+}, \triangleleft^{-}\right)$is the glb of the set of the pre-fixed points of $\Psi$. We get $\triangleleft^{+} \subseteq \triangleleft^{-}$, by definition of $\sqsubseteq$.

We prove the converse, $\triangleleft^{-} \subseteq \triangleleft^{+}$. For any $\ell \in \mathbb{N}$ and $v \in \mathrm{P}(\mathcal{D})$, we define the vector $\left.v\right|_{\ell} \in \mathrm{P}(\mathcal{D})$ as follows: $\left(\left.v\right|_{\ell}\right)_{d}:=v_{d}$, if $d \in\left|\mathcal{D}_{\ell}\right|$, otherwise $\left(\left.v\right|_{\ell}\right)_{d}:=0$. Notice $\left.v\right|_{\ell}$ is the canonical extension to $\mathcal{D}$ of the approximation $\left.v\right|_{\left|\mathcal{D}_{\ell}\right|}$ in $\mathrm{P}\left(\mathcal{D}_{\ell}\right)$ (Figure 3). Also, $\left(\left.v\right|_{\ell}\right)_{\ell \in \mathbb{N}}$ is an increasing sequence converging to its lub $v$. We will prove:

$(*) \forall \ell \in \mathbb{N}, \forall v \in \mathrm{P}(\mathcal{D}),(v, M) \in \triangleleft^{-} \Rightarrow\left(\left.v\right|_{\ell}, M\right) \in \triangleleft^{+}$.

In fact, $(*)$ implies $\triangleleft^{-} \subseteq \triangleleft^{+}$: if $(v, M) \in \triangleleft^{-}$, then by $(*)$ for every $\ell,\left(\left.v\right|_{\ell}, M\right) \in \triangleleft^{+}=\Phi\left(\triangleleft^{-}\right)$. Now, by Lemma 15 applied to $\triangleleft^{-},\left(\left.\sup _{\ell} v\right|_{\ell}, M\right)=(v, M) \in \triangleleft^{+}$.

The proof of $(*)$ is by induction on $\ell$. For any $v$, $\left.v\right|_{0}=\mathbf{0}$, so the claim follows by $\triangleleft^{+}=\Phi\left(\triangleleft^{-}\right)$and Lemma 15. Otherwise, let $(v, M) \in \triangleleft^{-}$, we prove that $\left(\left.v\right|_{\ell+1}, M\right) \in \triangleleft^{+}=\Phi\left(\triangleleft^{-}\right)$, that is, by Definition 12, for any $u \in \mathrm{P}\left(\mathcal{D}^{\mathbb{N}}\right)$, any number $n$ and $N_{0}, \ldots, N_{n-1}$ such that $\forall i<n,\left(\pi_{i}(u), N_{i}\right) \in \triangleleft^{-}$and $\forall i \geq n, \pi_{i}(u)=e_{\star}$, we prove $\left\langle\left. v\right|_{\ell+1}, u^{!}\right\rangle \leq \sum_{H \in \text { hnf }} \operatorname{Red}_{\left(M N_{0} \ldots N_{n-1}\right), H}^{\infty}$. First, since $(v, M) \in \triangleleft^{-}=\Phi\left(\triangleleft^{+}\right)$, by the downward closedness of $\Phi\left(\triangleleft^{+}\right)_{M}$ (Lemma 15), $\left(\left.v\right|_{\ell+1}, M\right) \in \Phi\left(\triangleleft^{+}\right)$. Then by induction hypothesis, for all $i<n$, since $\left(\pi_{i}(u), N_{i}\right) \in \triangleleft^{-}$, we have $\left(\left.\pi_{i}(u)\right|_{\ell}, N_{i}\right) \in \triangleleft^{+}$. Let $\bar{u} \in \mathrm{P}\left(\mathcal{D}^{\mathbb{N}}\right)$ be the stack of the first $n$-terms of $u$ restricted to $\mathcal{D}_{\ell}$, defined as $\pi_{i}(\bar{u})=\left.\pi_{i}(u)\right|_{\ell}$ for $i<n$, and $\pi_{i}(\bar{u})=e_{\star}$ for $i \geq n$. Finally, notice that $\left.v\right|_{\ell+1}$ is non-zero only on those $d$ that are in the web of $\mathcal{D}_{\ell+1}$, and for such $d, u_{d}^{!}=\bar{u}_{d}^{!}$. We infer: $\left\langle\left. v\right|_{\ell+1}, u^{!}\right\rangle=$ $\sum_{d \in|\mathcal{D}|}\left(\left.v\right|_{\ell+1}\right)_{d}\left(u^{!}\right)_{d}=\sum_{d \in\left|\mathcal{D}_{\ell+1}\right|}\left(\left.v\right|_{\ell+1}\right)_{d}\left(u^{!}\right)_{d}=$ $\sum_{d \in|\mathcal{D}|}\left(\left.v\right|_{\ell+1}\right)_{d}\left(\bar{u}^{!}\right)_{d}=\left\langle\left. v\right|_{\ell+1}, \bar{u}\right\rangle$. Finally, we conclude since $\left\langle\left. v\right|_{\ell+1}, \bar{u}\right\rangle \leq \sum_{H \in \text { hnf }} \operatorname{Red}_{\left(M N_{0} \ldots N_{n-1}\right), H}^{\infty}$, by the hypothesis that $\left(\left.v\right|_{\ell+1}, M\right) \in \triangleleft^{-}=\Phi\left(\triangleleft^{+}\right)$and for every $i<n,\left(\pi_{i}(\bar{u}), N_{i}\right) \in \triangleleft^{+}$.

We now prove $(\llbracket M \rrbracket, M) \in \triangleleft=\Phi(\triangleleft)$ for every closed term $M$. After some preliminary lemmas, Lemma 21 achieves the result by structural induction on $M$. Lemma 19 is noteworthy, stating that $\triangleleft$ is a fixed point also for the operation on relations corresponding with the $\mathcal{X} \mapsto \mathcal{X} \Rightarrow \mathcal{X}$ operation on PCS, having so a further analogy with $\mathcal{D}$.

Lemma 17. For any relation $R$, and vector $v \in \mathrm{P}(\mathcal{D})$, $(v, *) \in \Phi(R)$. In particular $\left(e_{\star}, *\right) \in \triangleleft$.

Proof: Notice that $\sum_{H \in \operatorname{hnf}} \operatorname{Red}_{* N_{0} \ldots N_{n-1}, H}^{\infty}=1$ for any terms $N_{0}, \ldots, N_{n-1}$. Then $(v, *) \in \Phi(R)$, since $\left\langle v, u^{!}\right\rangle \leq 1$ for any $u \in \mathcal{D}^{\mathbb{N}}$, by definition of orthogonality. The last part of the lemma is an immediate consequence of $\triangleleft=\Phi(\triangleleft)$ and the remark that $e_{\star} \in \mathrm{P}(\mathcal{D})$.

Lemma 18. If $M \stackrel{1}{\rightarrow} M^{\prime}$, then $(v, M) \in \triangleleft$ iff $\left(v, M^{\prime}\right) \in \triangleleft$.

Proof: By Lemma 2, item 3 and by $\triangleleft=\Phi(\triangleleft)$.

Lemma 19. Let $(v, M),(r, L) \in \triangleleft$, then $\left(p v+(1-p) r, M+{ }_{p}\right.$ $L) \in \triangleleft$.

Proof: Take $u \in \mathrm{P}\left(\mathcal{D}^{\mathbb{N}}\right), n \in \mathbb{N}, N_{0}, \ldots, N_{n-1} \in$ $\Lambda_{0}^{+}$such that $\forall i<n,\left(\pi_{i}(u), N_{i}\right) \in R$ and $\forall i \geq$ $n, \pi_{i}(u)=e_{\star}$. By linearity, $\left\langle p v+(1-p) r, u^{!}\right\rangle=p\left\langle v, u^{!}\right\rangle$ $+(1-p)\left\langle r, u^{!}\right\rangle$. By the hypothesis $(v, M),(r, L) \in \triangleleft=$ $\phi(\triangleleft)$, we have $\left\langle v, u^{!}\right\rangle \leq \sum_{H \in \mathrm{hnf}} \operatorname{Red}_{M N_{0} \ldots N_{n-1}, H}^{\infty}$ and $\left\langle r, u^{!}\right\rangle \leq \sum_{H \in \mathrm{hnf}} \operatorname{Red}_{L N_{0} \ldots N_{n-1}, H}^{\infty}$. We conclude, since for every $H, p \operatorname{Red}_{M N_{0} \ldots N_{n-1}, H}^{\infty}+(1-p) \operatorname{Red}_{L N_{0} \ldots N_{n-1}, H}^{\infty}=$ $\operatorname{Red}_{\left(M+{ }_{p} L\right) N_{0} \ldots N_{n-1}, H}^{\infty}$ (Lemma 2, item 2). 
Lemma 20. $(v, M) \in \triangleleft$ if, and only if, $\forall(r, L) \in \triangleleft$, $(\operatorname{app}(v)(r), M L) \in \triangleleft$.

Proof: Suppose that $(v, M) \in \triangleleft$ and $(r, L) \in$ $\triangleleft$, we prove $(\operatorname{app}(v)(r), M L) \in \Phi(\triangleleft)=\triangleleft$. Take $u \in \mathrm{P}\left(\mathcal{D}^{\mathbb{N}}\right), n \in \mathbb{N}, N_{0}, \ldots, N_{n-1} \quad$ s.t. $\forall i<$ $n,\left(\pi_{i}(u), N_{i}\right) \in \triangleleft$ and $\forall i \geq n, \pi_{i}(u)=e_{\star}$. By Lemma 8, $\left\langle\operatorname{app}(v)(r), u^{!}\right\rangle=\left\langle v,(r:: u)^{!}\right\rangle$. By $(v, M) \in \triangleleft=$ $\phi(\triangleleft),\left\langle v,(r:: u)^{!}\right\rangle \leq \sum_{H \in \text { hnf }} \operatorname{Red}_{M L N_{0} \ldots N_{n-1}, H}^{\infty}$. We conclude $(\operatorname{app}(v)(r), M L) \in \Phi(\triangleleft)$.

Conversely, suppose that for every $(r, L) \in \triangleleft$ we have $(\operatorname{app}(v)(r), M L) \in \triangleleft$, we prove that $(v, M) \in \Phi(\triangleleft)=$ $\triangleleft$. Take $u \in \mathrm{P}\left(\mathcal{D}^{\mathbb{N}}\right), n \in \mathbb{N}, N_{0}, \ldots, N_{n-1} \quad$ s.t. $\forall i<$ $n,\left(\pi_{i}(u), N_{i}\right) \in \triangleleft$ and $\forall i \geq n, \pi_{i}(u)=e_{\star}$. We split in two cases.

If $n=0$, then $u=e_{\vec{\star}}=e_{\star}:: e_{\vec{\star}}$. So Lemma 8 gives $\left\langle v,\left(e_{\vec{\star}}\right)^{!}\right\rangle=\left\langle\operatorname{app}(v)\left(e_{\star}\right),\left(e_{\vec{\star}}\right)^{!}\right\rangle$. By Lemma 17, $\left(e_{\star}, *\right) \in \triangleleft$, hence by hypothesis, $\left(\operatorname{app}(v)\left(e_{\star}\right), M *\right) \in \triangleleft=\Phi(\triangleleft)$. Applying Definition 12 to $n=0$ and $e_{\vec{x}} \in \mathrm{P}\left(\mathcal{D}^{\mathbb{N}}\right)$, we get $\left\langle\operatorname{app}(v)\left(e_{\star}\right),\left(e_{\vec{\star}}\right)^{!}\right\rangle \leq \sum_{H \in \mathrm{hnf}} \operatorname{Red}_{M *, H}^{\infty}=\sum_{H \in \mathrm{hnf}} \operatorname{Red}_{M, H}^{\infty}$ by Lemma 2 , item 4 .

If $n>0$, then let $\bar{u} \in \mathrm{P}\left(\mathcal{D}^{\mathbb{N}}\right)$ such that $u=$ $\pi_{0}(u):: \bar{u} \quad\left(\right.$ set $\left.\pi_{i}(\bar{u})=\pi_{i+1}(u)\right)$. Lemma 8 gives $\left\langle v, u^{!}\right\rangle=\left\langle\operatorname{app}(v)\left(\pi_{0}(u)\right), \bar{u}^{l}\right\rangle$. As $\left(\operatorname{app}(v)\left(\pi_{0}(u)\right), M N_{0}\right) \in \triangleleft$, we get $\left\langle\operatorname{app}(v)\left(\pi_{0}(u)\right), \bar{u}^{!}\right\rangle \leq \sum_{H \in \operatorname{hnf}} \operatorname{Red}_{\left(M N_{0}\right) N_{1} \ldots N_{n-1}, H}^{\infty}$.

Lemma 21. Let $M \in \Lambda^{+}, \Gamma=\left(y_{0}, \ldots, y_{n-1}\right) \supseteq \mathrm{FV}(M)$, $\left(u_{0}, N_{0}\right), \ldots,\left(u_{n-1}, N_{n-1}\right) \in \triangleleft$. We have:

$$
\llbracket M \rrbracket^{\Gamma}\left(u_{0}, \ldots, u_{n-1}\right) \triangleleft M\left\{N_{0} / y_{0}, \ldots, N_{n-1} / y_{n-1}\right\} .
$$

Proof: By structural induction on $M$. In the sequel, we will denote by $\llbracket M \rrbracket^{\Gamma} \vec{u}$ the vector $\llbracket M \rrbracket^{\Gamma}\left(u_{0}, \ldots, u_{n-1}\right) \quad$ in $\mathrm{P}(\mathcal{D})$, and by $M\{\vec{N} / \vec{y}\}$ the closed term $M\left\{N_{0} / y_{0}, \ldots, N_{n-1} / y_{n-1}\right\}$.

If $M=y_{i}$, the claim follows by the hypothesis $u_{i} \triangleleft N_{i}$.

If $M=*$, the claim follows by Lemma 17 .

If $M=\lambda z . L$, by induction hypothesis, for every $(r, R) \in$ $\triangleleft, \llbracket L \rrbracket^{z, \Gamma}\left(r, u_{0}, \ldots, u_{n-1}\right) \triangleleft L\{R / z, \vec{N} / \vec{y}\}$. By definition of $\llbracket \rrbracket, \llbracket L \rrbracket^{z, \Gamma}\left(r, u_{0}, \ldots, u_{n-1}\right)=\operatorname{app}\left(\llbracket M \rrbracket^{\Gamma} \vec{u}\right)(r)$, and by Lemma 2, item $1, M\{\vec{N} / \vec{y}\} R \stackrel{1}{\rightarrow} L\{R / z, \vec{N} / \vec{y}\}$, then by Lemma 18, $\operatorname{app}\left(\llbracket M \rrbracket_{\Gamma} \vec{u}\right)(r) \triangleleft M\{\vec{N} / \vec{y}\} R$. Since this is true for any $(r, R) \in \triangleleft$, Lemma 20 allows to conclude $\llbracket M \rrbracket_{\Gamma}(\vec{u}) \triangleleft M\{\vec{N} / \vec{y}\}$.

If $M=L_{1} L_{2}$, by induction hypothesis we have $\llbracket L_{i} \rrbracket^{\Gamma} \vec{u} \triangleleft L_{i}\{\vec{N} / \vec{y}\}$, for $i=1,2$. By definition of $\llbracket \rrbracket$, $\llbracket M \rrbracket^{\Gamma} \vec{u}=\operatorname{app}\left(\llbracket L_{1} \rrbracket^{\Gamma} \vec{u}\right)\left(\llbracket L_{2} \rrbracket^{\Gamma} \vec{u}\right)$, and by Lemma 20 , $\operatorname{app}\left(\llbracket L_{1} \rrbracket^{\Gamma} \vec{u}\right)\left(\llbracket L_{2} \rrbracket^{\Gamma} \vec{u}\right) \triangleleft L_{1}\{\vec{N} / \vec{y}\} L_{2}\{\vec{N} / \vec{y}\}=M\{\vec{N} / \vec{y}\}$.

If $M=L_{1}+{ }_{p} L_{2}$, by induction hypothesis $\llbracket L_{i} \rrbracket^{\Gamma}(\vec{u}) \triangleleft$ $L_{i}\{\vec{N} / \vec{y}\}$, for $i=1,2$. By definition of $\llbracket \rrbracket$, $\llbracket M \rrbracket^{\Gamma}(\vec{u})=$ $p \llbracket L_{1} \rrbracket^{\Gamma}(\vec{u})+(1-p) \llbracket L_{2} \rrbracket^{\Gamma}(\vec{u})$, we conclude by Lemma 19 .

\section{B. Main Results}

Theorem 22. For any $M \in \Lambda_{0}^{+}$, we have

$$
\sum_{d \in\left|\mathcal{D}_{2}\right|} \llbracket M \rrbracket_{d}=\sum_{H \in \mathrm{hnf}} \operatorname{Red}_{M, H}^{\infty} .
$$

Proof: Proposition 11 gives one inequality. For the converse, Lemma 21 gives $(\llbracket M \rrbracket, M) \in \triangleleft$, so by Proposition $16,(\llbracket M \rrbracket, M) \in \Phi(\triangleleft)$. Hence, by definition of $\Phi$, $\left\langle\llbracket M \rrbracket, e_{\overrightarrow{\mathrm{x}}}^{\vdots}\right\rangle \leq \sum_{H \in \mathrm{hnf}} \operatorname{Red}_{M, H}^{\infty}$. We conclude since by Lemma $9,\left\langle\llbracket M \rrbracket, e_{\vec{\not}}^{!}\right\rangle=\sum_{d \in\left|\mathcal{D}_{2}\right|} \llbracket M \rrbracket_{d}$.

A corollary of the adequacy theorem is the soundness of the order $\leq$ on vectors (Equation (4)) with respect to a suitable operational pre-order $\preceq$ on terms.

Definition 23. For any terms $M, N$, we define $M \preceq N$ whenever for every context $C[\cdot], \quad \sum_{H \in \mathrm{hnf}} \operatorname{Red}_{C[M], H}^{\infty} \leq$ $\sum_{H \in \mathrm{hnf}} \operatorname{Red}_{C[N], H}^{\infty}$.

Lemma 24. The interpretation $\llbracket \cdot \rrbracket$ is context closed: if $\llbracket M \rrbracket^{\Gamma} \leq \llbracket N \rrbracket^{\Gamma}$, then $\forall C[\cdot], \llbracket C[M] \rrbracket^{\Gamma} \leq \llbracket C[N] \rrbracket^{\Gamma}$.

Proof: Easy structural induction on $C[\cdot]$.

Corollary 25. Let $M, N \in \Lambda^{+}$and $\Gamma \supseteq \mathrm{FV}(M) \cup \mathrm{FV}(N)$, then $\llbracket M \rrbracket^{\Gamma} \leq \llbracket N \rrbracket^{\Gamma}$ entails $M \preceq N$.

Proof: By Lemma 24 and Theorem 22.

\section{CONCLUSION}

We studied a reflexive object $\mathcal{D}$ in the category $\mathbf{P c o h}_{!}$of probabilistic coherence spaces and entire functions. The object $\mathcal{D}$ has been introduced by the first author and Danos as the limit of a chain $\left\{\mathcal{D}_{\ell}\right\}_{\ell \in \mathbb{N}}$ of PCSs [DE11]. We proved the adequacy of $\mathcal{D}$ with respect to a probabilistic extension $\Lambda^{+}$of the pure $\lambda$-calculus: the probability that a closed term of $\Lambda^{+}$ reaches a head normal form is equal to the sum of the values of its interpretation when projected to $\mathcal{D}_{2}$.

An obvious issue arising from our results is whether the converse of Corollary 25 holds, i.e. whether probabilistic coherence spaces are fully abstract with respect to the observational pre-order given in Definition 23. Indeed, a slight modification of the above proofs gives that projecting the interpretations of the terms to $\mathcal{D}_{1}$ gives the probability that these terms reduce to closed head normal forms having $*$ in head position. We wonder whether one can state a general result precisely relating each PCS in the chain $\left\{\mathcal{D}_{\ell}\right\}_{\ell \in \mathbb{N}}$ with a suitable hierarchy of observational pre-orders.

Also, we plan to investigate the expressive power of $\Lambda^{+}$ (hence of PCSs), namely with respect to the stochastic processes. The fact that $\Lambda^{+}$extends pure $\lambda$-calculus suggests that it can model a wide class of computational phenomena, also in a probabilistic setting. For example, we conjecture that every finite stochastic chain can be expressed by a closed term of $\Lambda^{+}$. This deserves further work.

\section{ACKNOWLEDGMENT.}

This work has been partly supported by the French ANR project ANR-08-BLAN-0324 Curry-Howard for Concurrency (CHOCO) and the chaire CNRS "Logique linéaire et calcul".

\section{REFERENCES}

[Bar84] Henk Barendregt. The Lambda-Calculus, its Syntax and Semantics. Stud. Log. F. Math., vol. 103. North-Holland, 1984. 
[BEM] Antonio Bucciarelli, Thomas Ehrhard, and Giulio Manzonetto. Not Enough Points Is Enough. CSL, Lecture Notes in Comput. Sci. 4646:298-312, 2007.

[DE11] Vincent Danos and Thomas Ehrhard. Probabilistic coherence spaces as a model of higher-order probabilistic computation. Inform. Comput., 2011.

[Ehr05] T. Ehrhard. Finiteness spaces. Math. Structures Comput. Sci., 15(04):615-646, 2005.

[Gir87] Jean-Yves Girard. Linear logic. Th. Comp. Sc., 50:1-102, 1987.

[Gir88] Jean-Yves Girard. Normal functors, power series and lambdacalculus. Ann. Pure Appl. Logic, 37(2):129-177, 1988.

[Gir04] Jean-Yves Girard. Between logic and quantic: a tract. In Thomas Ehrhard, Jean-Yves Girard, Paul Ruet, and Philip Scott, editors, Linear Logic in Computer Science, volume 316 of London Math. Soc. Lecture Note Ser. CUP, 2004.

[Hyl76] Martin Hyland. A syntactic characterization of the equality in some models for the lambda calculus. J. London Math. Soc, 12:361-370, 1976.

[Jon89] Claire Jones. Probabilistic Non-Determinism. PhD thesis, University of Edimburgh, 1989.

[JP89] Claire Jones and Gordon Plotkin. A probabilistic powerdomains of evaluation. In Proceedings of the 4th Annual IEEE Symposium on Logic in Computer Science. IEEE Computer Society, 1989.

[Pit] A. M. Pitts. Computational adequacy via 'mixed' inductive definitions. MFPS, Lecture Notes in Comput. Sci. 802:72-82, 1994.

[Plo77] Gordon D. Plotkin. LCF considered as a programming language. Theor. Comput. Sci., 5(3):225-255, 1977.

[Rey] John C. Reynolds. On the relation between direct and continuation semantics. ICALP, Lecture Notes in Comput. Sci., 14:141-156, 1974.

[Tar55] Alfred Tarski. A lattice theoretical fixpoint theorem and its applications. Pacific J. Math., 5:285-309, 1955 